\title{
Integración florística de las algas marinas de la costa sur de Jalisco, México
}

\author{
Floristic integration of the benthic marine algae from the southern coast of Jalisco, Mexico
}

\author{
A. Catalina Mendoza-González ${ }^{1 *}$, Luz Elena Mateo-Cid ${ }^{1}$ y Citlali Galicia-García ${ }^{2}$ \\ ${ }^{1}$ Departamento de Botánica, Escuela Nacional de Ciencias Biológicas. Carpio y Plan de Ayala, Col. Santo Tomás, 11340 México, D.F., México. \\ ${ }^{2}$ Departamento de Biología, Instituto Tecnológico del Mar. 94290 Boca del Río, Veracruz, México. \\ *Correspondencia: am7124@gmail.com
}

\begin{abstract}
Resumen. Se presentan los resultados obtenidos en un estudio sobre las algas marinas bentónicas en 7 localidades de la costa sur de Jalisco, México. Se determinó la presencia de 165 especies de algas marinas. Se identificaron 13 especies de Cyanophyta, 97 Rhodophyta, 28 Chlorophyta y 27 taxa de Heteroconthophyta. Cada especie se acompaña con datos sobre su distribución en el área de estudio, estado reproductivo, nivel de marea, hábitat, observaciones, epifitismo y número de herbario o de colecta. Se comparó la riqueza específica entre la estación climática de lluvias y la de secas, durante 1992,1993, 1997, 2000 y 2003. La división Rhodophyta dominó en términos de diversidad en relación con las 3 divisiones restantes. La ficoflora de la costa sur de Jalisco es de afinidad tropical y más diversa en la época de secas.

Palabras clave: Cyanophyta, Rhodophyta, Heteroconthophyta, Chlorophyta, riqueza específica.

Abstract. We present results on the study on benthic marine algae in seven localities from the southern coast of Jalisco. We report 165 species: 13 Cyanophyta, 97 Rhodophyta, 28 Chlorophyta and 27 Heteroconthophyta. Each species includes data on its distribution, reproductive stages, tidal level, facies, epiphytism and herbarium's number. Species diversity was compared for 2 different climatic seasons during 1992, 1993, 1997, 2000 and 2003. The Rhodophyta are dominant in terms of diversity in relation to the other groups. The algal flora of the south coast of Jalisco is tropical and the greatest diversity was found during dry season.
\end{abstract}

Key words: Cyanophyta, Rhodophyta, Heterocontophyta, Chlorophyta, species diversity.

\section{Introducción}

La ubicación y fisiografía del territorio de la costa del Pacífico de México, presenta características notables. Las regiones mejor conocidas ficoflorísticamente son el golfo de California y el Pacífico de Baja California (Pedroche et al., 2005, 2008; Norris, 2010) En contraste, la región menos estudiada es la tropical, en la cual está incluida la porción norte del Pacífico tropical de México, cuyos límites se encuentran en la frontera entre Nayarit y Sinaloa al norte, y en la frontera entre los estados de Jalisco y Colima al sur (Serviere Zaragoza et al., 1993). En esta región se localiza la zona objeto del presente estudio: la costa sur de Jalisco.

Los primeros registros de algas marinas de la costa sur de Jalisco fueron los de Dawson en Tenacatita en 1949. Posteriormente, éstos se enriquecieron con otros trabajos de Dawson $(1951,1953 a, 1954,1960,1961)$ y de Chávez-

Recibido: 05 octubre 2009; aceptado: 19 abril 2010
Barrera (1972, 1980). El primer estudio ficoflorístico de la región sur del estado de Jalisco fue realizado por Pedroche y González-González (1981), seguido por el de EncisoPadilla et al. (1995) y el de Águila-Ramírez et al. (1998). Además se tienen los trabajos de Mendoza-González y Mateo-Cid (1992), Serviere-Zaragoza et al. (1993, 1998), León-Álvarez et al. (1997), Pedroche et al. (2005) y Rodríguez et al. (2008), en los que se registran varias especies de algas marinas para el litoral sur de Jalisco. En los citados estudios se mencionan 77 especies de algas marinas para la costa sur de Jalisco, a los que se incorporan los nuevos registros del presente trabajo y una integración de la composición florística de las algas de la región y su variación estacional en las épocas de lluvias y secas.

\section{Materiales y métodos}

Las muestras ficológicas se obtuvieron mediante 4 muestreos en la estación climática de lluvias (julio, 1992; septiembre, 1998, 2000, 2003 y octubre, 1992, 
2000) y en la de secas (noviembre, 1992, 1993, 1998, 2000 y 2003). Las algas fueron recolectadas a mano en el nivel intermareal con ayuda de espátulas y navajas de campo. Las muestras se fijaron en formaldehído a $4 \%$ en agua de mar. El material fue procesado y depositado en el herbario ENCB. Para la descalcificación de taxa de la familia Corallinaceae y Liagoraceae se utilizó $\mathrm{HNO}_{3}$ $0.6 \mathrm{~N}$. La determinación del material ficológico se llevó a cabo de acuerdo con Taylor (1945,1960), Dawson (1953a, b, 1954, 1960, 1961, 1962, 1963a y 1963b), Hollenberg (1961), Abbott y Hollenberg (1976), Norris y Johansen (1981), Cho et al. (2008) y Anagnostidis y Komáreck (1988). La secuencia de la lista florística sigue el orden propuesto por Wynne (2005) y Pedroche et al. (2005, 2008 ). La actualización nomenclatural se basó en Guiry y Guiry (2008). Cada especie se acompaña con datos sobre distribución en el área de estudio, estado reproductivo, nivel de marea, el sustrato, hábitat, epifitismo y número de herbario o de recolecta.

Zona de estudio y localidades de muestreo (Fig. 1). El estado de Jalisco se encuentra ubicado en el occidente de la República Mexicana, entre los $18^{\circ} 55^{\prime}$ y $22^{\circ} 45^{\prime} \mathrm{N}$ y $10^{\circ} 28^{\prime}$ y $105^{\circ} 43^{\prime} \mathrm{O}$. La costa del estado forma parte del Pacífico tropical mexicano (PTM), con longitud de 250 $\mathrm{km}$ en dirección NO-SE. Por su topografía se divide en 3 partes: la primera abarca desde la desembocadura del río Cihuatlán hasta Chamela, la segunda de punta Férula al valle de Tomatlán y la tercera de Tehualmixtle a Puerto Vallarta en bahía de Banderas (Galicia-García, 1998).

La zona de estudio se encuentra en la primera de las regiones señaladas e incluye los municipios de la Huerta y Cihuatlán. Desde bahía de Chamela hasta barra de Navidad, la costa presenta una sucesión de acantilados rocosos que tienen de 15 a $30 \mathrm{~m}$ de altura y bajos con pequeñas playas de arena; las rocas son de origen volcánico (GaliciaGarcía, 1998).

El clima de la región es de tipo Awo (w) i, cálido húmedo con lluvias de verano y poca oscilación térmica a lo largo del año, la temporada de lluvias se presenta de junio a octubre y la de secas de noviembre a mayo (García, 1980). Las mareas son semidiurnas con una amplitud de alrededor de 3 metros. El material biológico se obtuvo en 7 localidades: Chamela, Careyes, Tenacatita, La Manzanilla, Cuastecomate, Melaque y barra de Navidad.

Chamela es una bahía de aproximadamente $9 \mathrm{~km}$ de extensión, con amplia playa arenosa, por la cual, en dirección norte, se llega a una punta rocosa con acantilados y a una playa con piedras, guijarros y cantos rodados. Durante la bajamar se observa una plataforma rocosa con pozas de marea.

Careyes (Cala Careyitos) es una playa mixta, de pendiente pronunciada arena gruesa y formaciones rocosas en los extremos, donde existen canales de corriente y pozas intermareales.

Playa Tenacatita es una playa arenosa con numerosas rocas esparcidas en una área de aproximadamente $900 \mathrm{~m}$, con abundantes esqueletos de coral, al final de la cual se encuentra una zona rocosa expuesta.

La Manzanilla está ubicada en el límite SE de la bahía de Tenacatita. En los extremos de este lugar hay formaciones rocosas y acantilados, a partir de estos se forman plataformas rocosas con canales de corriente y durante la bajamar se forman pozas de marea. También existe una playa arenosa con esqueletos de coral.

Bahía de Cuastecomate se encuentra $2.5 \mathrm{~km}$ al oeste de San Patricio Melaque, tiene $1.5 \mathrm{~km}$ de amplitud, con playa arenosa al centro y de cantos rodados y rocas hacia los extremos, donde se forman pozas de marea.

Melaque se encuentra en el extremo NO de la bahía de Navidad. Tiene una playa de pendiente pronunciada; en el extremo de la playa se encuentra punta Melaque, zona rocosa con acantilados y peñascos.

Barra de Navidad está localizada a $1.5 \mathrm{~km}$. al SE de Melaque, este lugar tiene aproximadamente $3.5 \mathrm{~km}$. de amplitud y una playa arenosa con pendiente pronunciada. En el extremo sur de la bahía se encuentra una escollera. Las algas fueron recolectadas en las rocas de las escolleras y en el estero de Navidad, sobre los pilotes de madera del muelle.

\section{Resultados y discusión}

Flora y hábitat. En la costa sur de Jalisco, la cual es predominantemente rocosa, se recolectó un total de 165 especies de algas marinas bentónicas. Los ambientes donde habita la mayoría son plataformas rocosas, pozas de marea, sobre guijarros, cantos rodados, zonas de acantilados y canales de corriente. De estas especies, 13 corresponden a Cyanophyta (8.0\%); 97 a Rhodophyta (59.0\%); 27 a Heteroconthophyta (16.0\%) y 28 a Chlorophyta (17.0\%). Como puede observarse, la riqueza específica está dada principalmente por las algas rojas, organismos que por su cobertura y su diversidad son importantes en zonas tropicales y templadas, tienen un exitoso desarrollo como epizoicas, epilíticas y epífitas; sin embargo, por su escasa talla, algunas especies pasan inadvertidas. En este grupo las familias mejor representadas fueron: Corallinaceae con 22 especies, Rhodomelaceae con 17 y Ceramiaceae con 8 especies (Apéndice 1). De las Cyanophyta, que es un grupo con pocos registros previos, sólo fueron localizadas 13 especies; 10 son epífitas, y aún cuando no es un grupo muy diverso y numeroso, su importancia radica en el grado 


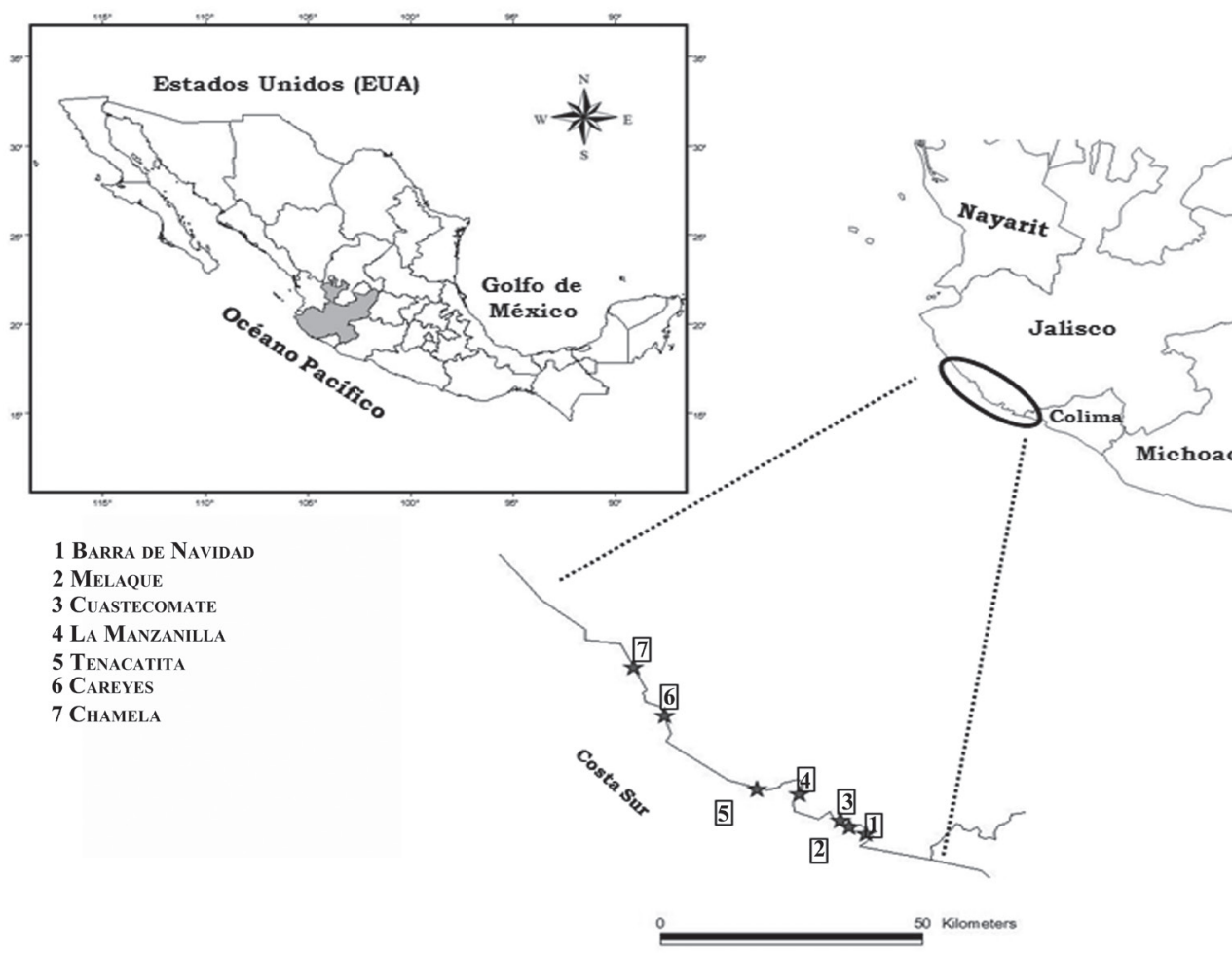

Figura 1. Ubicación del área de estudio y de las localidades de muestreo.

de epifitismo que establecen con sus hospederos, ya que algunas llegan a tener una cobertura de hasta un $90 \%$, lo que confiere una coloración verde azul a la superficie de las algas sobre las que se desarrollan.

En Heteroconthophyta se ubicaron 27 especies; la familia mejor representada fue Dictyotaceae con 10 taxa, todas de hábito epilítico. En Chlorophyta las familias más importantes fueron Cladophoraceae y Ulvaceae con 8 y 5 especies respectivamente; exceptuando 1 epizoica, el resto son especies epilíticas.

Biogeografia. La ficogeografía es una rama de la biogeografía que en función del tipo de tratamiento de los datos puede dividirse en 3 aproximaciones: ficogeografia descriptiva, relacionada con la obtención de datos básicos de distribución y establecimiento de modelos sencillos de distribución; ficogeografia narrativa que trata de explicar lo observado de una manera inductiva y ficogeografia analítica, que se ocupa de la distribución de las especies mediante la aplicación de un modelo hipotético-deductivo (Báez et al., 2004).

Para el estudio de la ficogeografia descriptiva, Feldmann (1937) propuso el índice R/P (número de especies de Rhodophyta entre número de especies de Phaeophyta) para clasificar la flora de una región determinada en función de un gradiente latitudinal. Así, un valor de la relación $\mathrm{R} / \mathrm{P}>4$ se suele encontrar en regiones tropicales, y un valor de $\mathrm{R} / \mathrm{P}<2$ corresponde con la ficoflora de regiones templadofrías. Cheney (1977) incluyó las Chlorophyta (C) en un índice análogo al comentado antes $(\mathrm{R}+\mathrm{C}) / \mathrm{P}$, y comprobó que valores de la relación $(\mathrm{R}+\mathrm{C}) / \mathrm{P}>6$ se obtienen en floras tropicales, en tanto que las floras de mares templados-fríos tienen valores $<3$.

Los resultados obtenidos en el presente estudio fueron analizados con los índices de Feldmann y Cheney y se compararon con los estudios de Mateo-Cid y MendozaGonzález (1991，1992, 2001) para Colima, Nayarit y Oaxaca, respectivamente; Mendoza-González y MateoCid (1998) de Guerrero, y Dreckmann et al. (2006) para el litoral de Chiapas. En el Cuadro 1, se muestran los valores obtenidos de los índices de Feldmann y Cheney para los 6 estados, y en él se observa que la ficoflora de la costa sur de Jalisco es similar a la ubicada en Colima, Guerrero y Oaxaca, estados que pertenecen al Pacifico Tropical de México y que de acuerdo con los valores obtenidos permiten establecer que la ficoflora de estos estados es mixta, con numerosos elementos tropicales como Amphiroa beauvoisii, A, misakiensis, Neogoniolithon trichotomum, Izziella orientalis, Dictyota crenulata, Asteronema breviarticulata, Ectocarpus siliculosus, Bachelotia antillarum, Chaetomorpha antennina y Ulva fasciata, 
Cuadro 1. Clasificación de la ficoflora con los índices de Feldmann y Cheney

\begin{tabular}{ccc}
\hline Estado & Índice Feldmann $(R / P)$ & Índice de Cheney $(R+C / P)$ \\
NAYARIT & 4.31 & 6.12 \\
COLIMA & 3.35 & 4.52 \\
GUERRERO & 3.7 & 4.44 \\
OAXACA & 4.31 & 5.0 \\
CHIAPAS & 3.63 & 5.63 \\
ESTE ESTUDIO & 3.59 & 4.63 \\
\hline
\end{tabular}

entre otros; sin embargo, también se localizaron especies de zonas templadas como Jania ungulata f. brevior, Lithophyllum decussatum, Callithamnion endovagum y Streblonema penetrale, entre otras. Sólo la ficoflora de la costa sur de Nayarit puede considerarse una flora tropical. Riqueza especifica. En la figura 2, se aprecia que la mayor riqueza específica se encontró durante la estación climática de secas, con137 taxa, y la menor en la de lluvias, con 79 especies. En la figura 3, se observa que las localidades 4, 5 y 7 que corresponden a La Manzanilla, Tenacatita y Chamela, respectivamente, se presentó el número más alto de especies en ambas estaciones climáticas, siendo Tenacatita la localidad con mayor riqueza específica de la costa sur de Jalisco, y Melaque la del menor número de especies (Fig. 3), sobre todo debido a que la playa arenosa presenta una pendiente muy pronunciada, ambiente difícil de colonizar por las algas ya que al accionar el oleaje sobre la arena, ésta se convierte en un material móvil y abrasivo que no permite la fijación de esporas o cigotos, por lo que la flora recolectada estuvo ubicada sobre las rocas de los acantilados.

De las 165 especies determinadas en este estudio para la costa sur de Jalisco, 70 representan nuevos registros para el área de estudio (NRJ) y 3 son registros nuevos para el litoral del Pacífico tropical (NRM) (Apéndice 1); por otro lado, 34 especies previamente registradas no se localizaron (Cuadro 2), quizá por los cambios en los ambientes donde habitan estas algas, donde actualmente existe una mayor

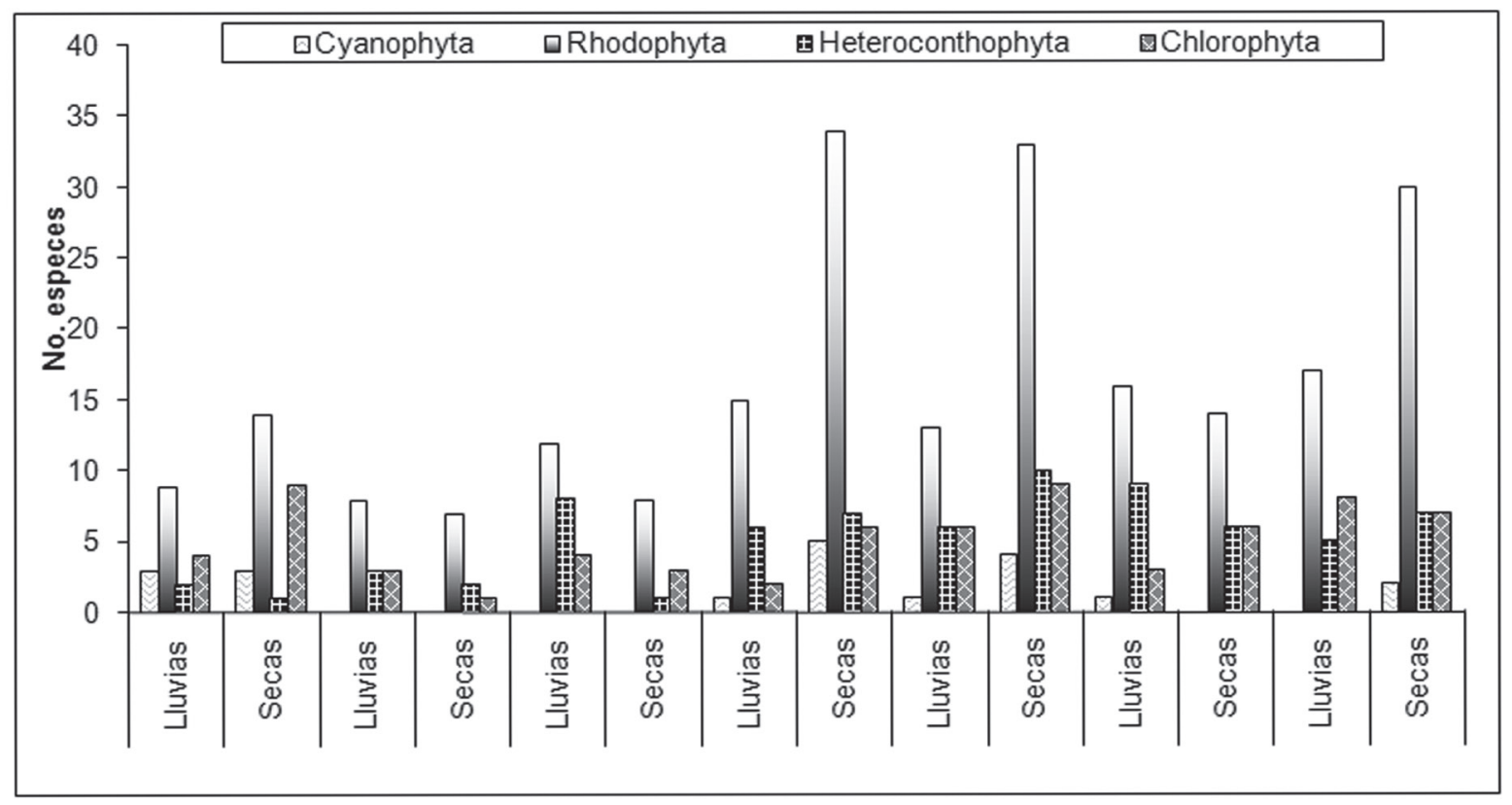

Figura 2. Número de especies por localidad y por estación climática. 
Cuadro 2. Citas anteriores de algas marinas para la costa sur de Jalisco, no ubicadas en este estudio

Grupo/Especie Referencia Actualización nomenclatural

\section{Cyanophyta}

1. Lyngbya birgei G. Smith

2. Plectonema wollei Farlow ex Gomont

3. Scytonema crispum (C.Agardh) Bornet

\section{Rhodophyta}

4. Galaxaura fastigiata Decaisne

5. Jania longiarthra E. Dawson

6. Gracilaria crispata Setchell et Gardner

7. G. tepocensis (E. Dawson) E. Dawson

8. G. textorii var. cuninghamii (Farlow) E. Dawson

9. Herposiphonia littoralis Hollenberg

10. Laurencia decidua E. Dawson

11. L. gardneri Hollenberg

12. Polysiphonia pacifica Hollenberg

13. Ahnfeltia plicata (Hudson) Fries

14. Jania tenella var. zacae E. Dawson

15. Gloiophloea confusa Setchell

16. Gloiopeltis furcata (Postels et Ruprecht) J. Agardh

\section{Heteroconthophyta}

17. Ralfsia pacifica Hollenberg

18. Sphacelaria tribuloides Meneghini

19. Dictyota bartayresii Lamouroux

20. D. ciliolata Sonder ex Kützing

21. D. flabellata (F.S. Collins) Setchell et Gardner

22. Padina pavonia Lamouroux

23. P. vickersiae Hoyt

24. Sargassum brandegeei Setchell et Gardner
Pedroche y González-González, 1981

Pedroche y González-González, 1981

Pedroche y González-González, 1981

Pedroche y González-González, 1981

Pedroche y González-González, 1981

Serviere-Zaragoza et al., 1993

Pedroche y González-González, 1981

Serviere-Zaragoza et al., 1993

Pedroche y González-González, 1981

Pedroche y González-González, 1981

Serviere-Zaragoza et al., 1993

Águila-Ramírez et al., 1998

Águila-Ramírez et al., 1998

Serviere-Zaragoza et al., 1993

Águila-Ramírez et al., 1998

Águila-Ramírez et al., 1998

Serviere-Zaragoza et al., 1993; Pedroche et al., 2008.

Águila-Ramírez et al., 1998

Pedroche y González-González, 1981; Serviere-Zaragoza et al., 1993

Pedroche y González-González, 1981

Enciso-Padilla et al., 1995

Chávez-Barrera 1980

Pedroche y González-González, 1981

Pedroche y González-González, 1981
Tricleocarpa cylindrica (J. Ellis et Solander) Lamouroux

Scinaia confusa (Setchell) Huisman

Dictyota barteyresiana Lamouroux

Padina pavonica (Linnaeus) Thivy

Padina gymnospora (Kützing) Sonder 
Cuadro 2. Continúa
Grupo/Especie
Referencia
Actualización nomenclatural

25. S. horridum Setchell et Gardner

\section{Chlorophyta}

26. Ulva californica Wille

27. U. dactylifera Setchell et Gardner

28. U. expansa (Setchell) Setchell et Gardner

29. U. lactuca Linnaeus

30. U. lobata (Kützing) Harvey

31. Enteromorpha salina Kützing

32. Bryopsis galapagensis W.R. Taylor

33. Caulerpa racemosa var uvifera

(C. Agardh) J. Agardh

34. Codium simulans Setchell et Gardner
Pedroche y González-González, 1981

Pedroche y González-González, 1981

Pedroche y González-González, 1981

Ulva taeniata (Setchell) Setchell et Gardner

Serviere-Zaragoza et al., 1993

Pedroche y González-González, 1981; Enciso-Padilla et al., 1995; Águila-Ramírez et al., 1998

Pedroche y González-González, 1981; Serviere-Zaragoza et al., 1993

Pedroche y González-González, 1981

Ulva prolifera O.F. Müller

Serviere-Zaragoza et al., 1993; Pedroche et al., 2005

Pedroche y González-González, 1981;

Serviere-Zaragoza et al., 1993; EncisoPadilla et al., 1995; Águila-Ramírez et al., 1998

Serviere-Zaragoza et al. 1998; Pedroche et al. 2005
Caulerpa racemosa (Forsskål) Lamouroux influencia humana que ha modificado la arquitectura del paisaje. La suma de los registros previos y los obtenidos en este estudio hace un total de 199 especies conocidas para la costa sur de Jalisco.

Considerando los estudios realizados previamente por Pedroche y González-González (1981); MendozaGonzález y Mateo- Cid (1992); Serviere-Zaragoza et al. (1993); Águila-Ramírez et al. (1998) y Pedroche et al. $(2005,2008)$ así como el del presente trabajo, el total de taxa para la costa de Jalisco es de 246, sin embargo es importante resaltar que la zona centro de la costa no ha sido estudiada, por lo que para conocer la composición ficoflorística en todo el litoral del estado, se requiere explorar esa región.
Las 246 especies conocidas para la costa de Jalisco revelan que esta flora es más diversa que la de Oaxaca, de la cual se han registrado193 taxa (Mateo-Cid y Mendoza González, 2001), la costa norte de Michoacán con 68, citadas por Dreckmann et al. (1990), y la costa sur de Nayarit donde se han registrado 119 (Mateo-Cid y Mendoza-González ,1992).

Composición. Las condiciones ecológicas indudablemente tienen influencia en la biología y estructura de las algas marinas, dichas condiciones influyen en la presencia o ausencia de las algas marinas en una estación en particular. Feldmann (1937) propuso una clasificación de tipos biológicos para las algas caracterizados por su tiempo de vida: 1) algas anuales, las cuales viven sólo en una 


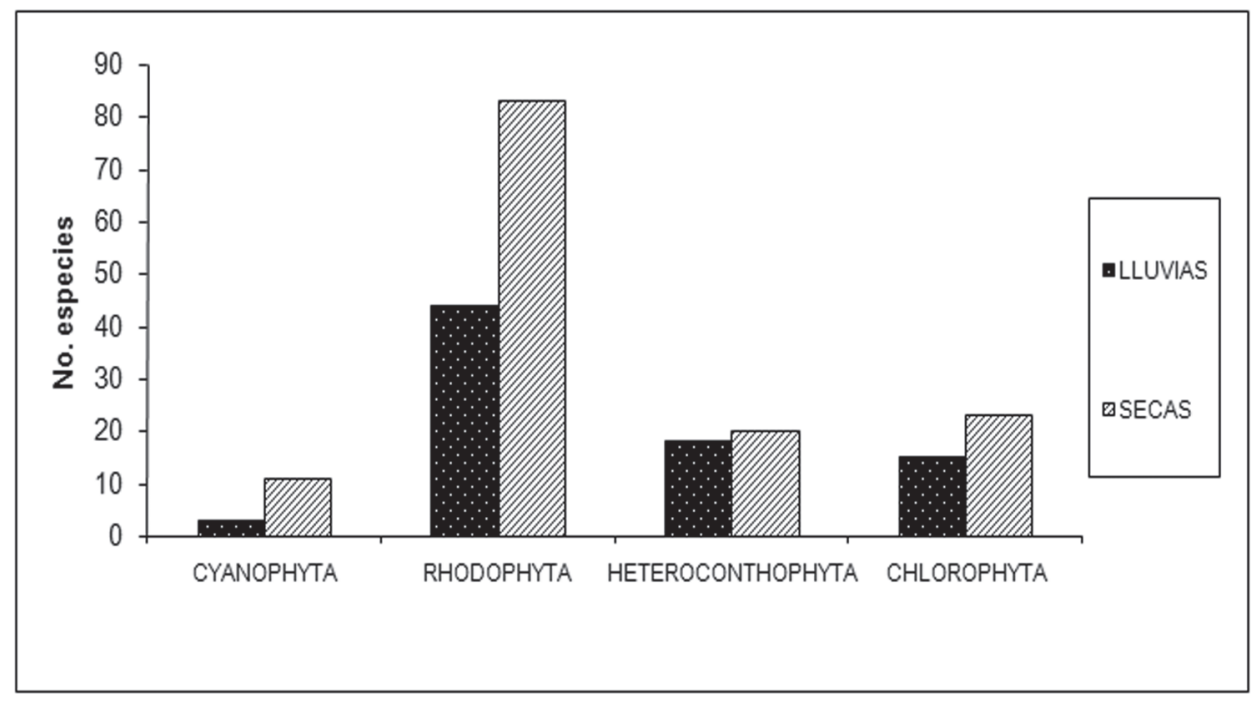

Figura 3. Número total de especies por estación climática.

estación o en la mayoría del año, y 2 )algas perennes, que son capaces de vivir varios años. En este estudio se localizaron principalmente algas anuales, como es el caso de las representantes de las familias Achrochaetiaceae, Liagoraceae, Ceramiaceae, Rhodomelaceae, Dasyaceae, Delesseriaceae, Ectocarpaceae, Dictyotaceae, Ulvaceae, Cladophoraceae y Codiaceae, y menos las perennes que están representadas por las familias Corallinaceae, Hapalidiaceae y Sargassaceae (Apéndice 1).

Como puede observarse en el Apéndice 1, las algas anuales dominan en la época de secas y disminuyen en número en la época de lluvias. Además, existe una marcada diferencia entre el número de taxa por localidades y por estación climática (Fig. 2). Así, en el grupo Cyanophyta, 2 especies son exclusivas de la época de lluvia y 9 de la época seca; Rhodophyta tiene un comportamiento similar, con 37 especies exclusivamente en secas y 11 en lluvias; por lo que respecta a Heteroconthophyta, se hallaron 4 especies en la época de secas y 3 en la de lluvias; finalmente, para Chlorophyta, 10 especies fueron exclusivas para secas y sólo 4 en lluvias. Como ejemplo de especies exclusivas están: Schizothrix calcicola, Calothrix crustacea, Microcystis zanardinii, Xenoccocus gilkeyae, Colaconema pectinatum, Halychrysis irregularis, Izziella orientalis, Galaxaura oblongata, Anotrichium tenue, Neosiphonia sphaerocarpa, Laurencia clarionensis, Hecatonema terminale, Streblonema penetrale, Sphacelaria rigidula y Cladophora vagabunda, taxa que pueden ubicarse en la categoría de algas anuales. En cuanto a las algas perennes, pueden ubicarse como exclusivas: Phymatolithon lenormandii, Hydrolithon onkodes, $H$. rupestre $\mathrm{y}$ Lithophyllum decussatum.
En la figura 2 se observa que La Manzanilla, Tenacatita y Chamela son las localidades donde se ubica el número más alto de especies en ambas estaciones climáticas, en este caso, las 3 localidades tienen como característica particular el ser playas donde domina el sustrato rocoso y por ende comparten entre ellas numerosas especies (Apéndice 1). Finalmente, sólo Amphiroa beauvoisii, Dermonema virens, Gelidium sclerophyllum, Chaetomorpha antennina $y$ Caulerpa peltata se ubicaron en 6 de las 7 localidades de muestreo y en ambas estaciones climáticas.

Reproducción. En la figura 4 se muestra que el mayor número de especies en fase reproductiva se localizó en la época de secas, siendo la fase tetraspórica la que superó tanto a las especies con fase gamética como a las especies con células de resistencia (heterocistos, gonidangios, acinetos). Estos datos permiten considerar que probablemente la generación tetraspórica tiene una mayor supervivencia con respecto a otras generaciones, así como a otros mecanismos de reproducción, como la propagación vegetativa o la apomeiosis (Ardito y Gómez, 2005).

En la figura 5 se observa que el $77.6 \%$ de las 165 especies ubicadas en este estudio presentaron algún tipo de estructura reproductora, siendo las algas Rhodophyta el grupo dominante, debido a que el $93.8 \%$ de sus representantes se encontró en alguna fase fértil y sólo el $6.19 \%$ se halló en estado vegetativo. La fase reproductiva dominante en Rhodophyta fue la tetraspórica, resultado que coincide con los obtenidos por Mateo-Cid y MendozaGonzález (1991, 1992, 2001) y Magahhães y Castro (2002); estos últimos autores mencionan que la dominancia de una fase reproductiva puede estar influenciada por cambios 


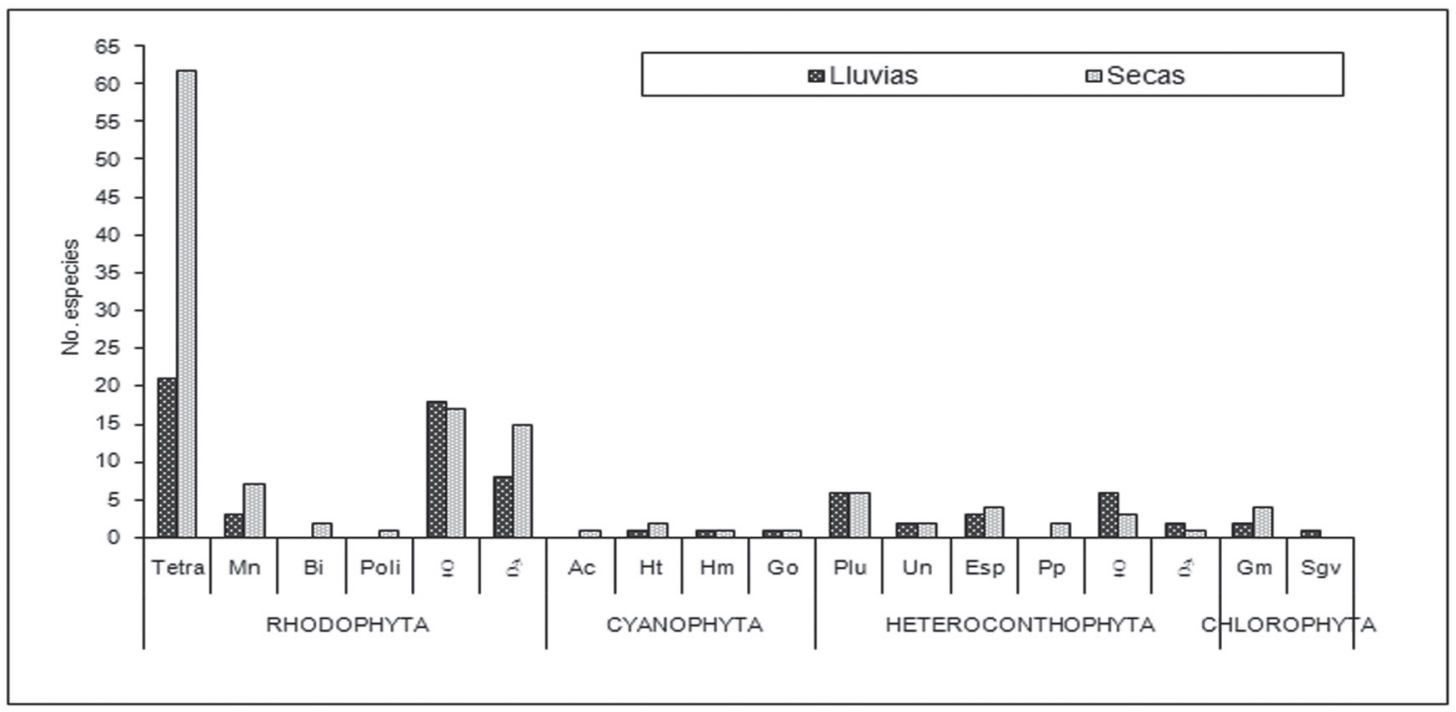

Figura 4. Estados reproductivos por división de algas.

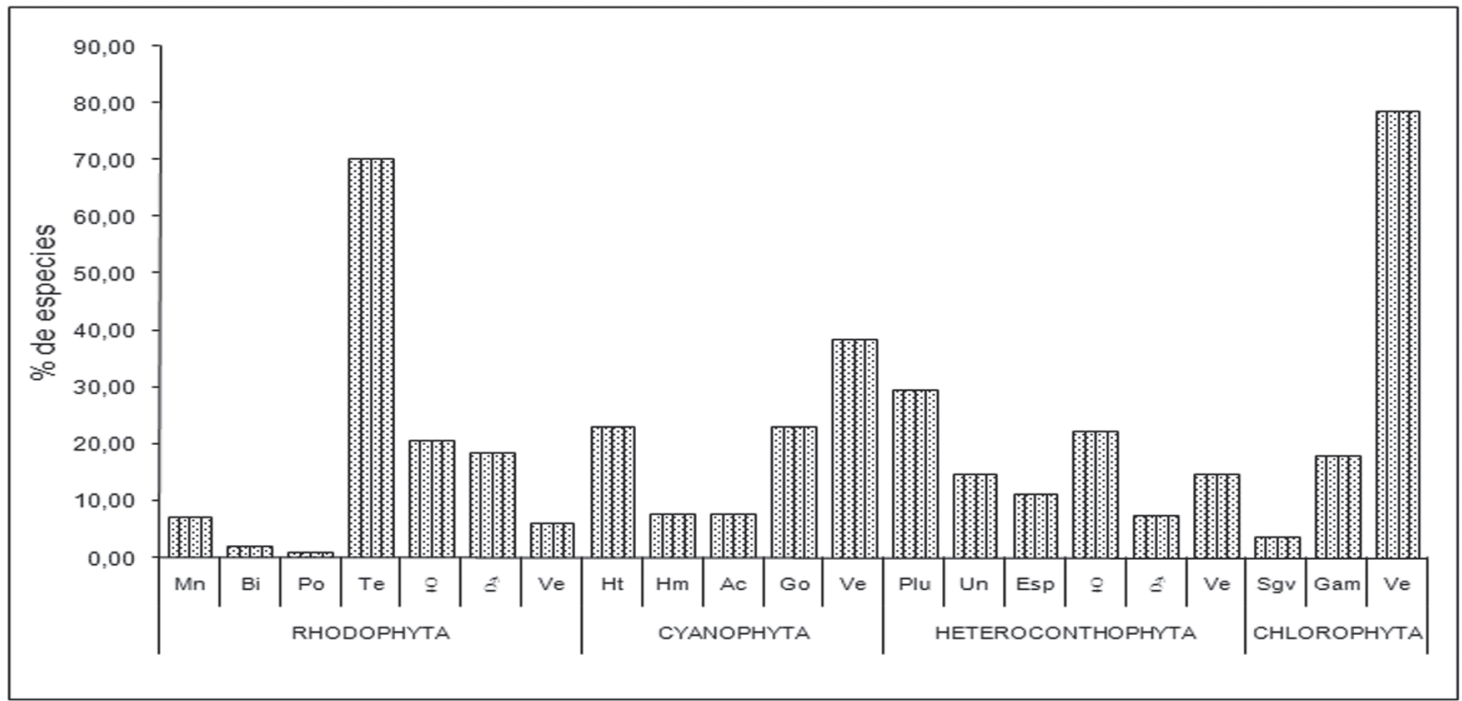

Figura 5. Composición porcentual de los estados reproductivos por división de algas.

temporales de factores ambientales locales. En el grupo Cyanophyta, el $61.54 \%$ presentó alguna fase reproductiva asexual, que es el tipo de reproducción común en estos organismos.

En cuanto a Heteroconthophyta, el $85.19 \%$ de sus representantes se encontró en reproducción; en este grupo se observaron estructuras pluriloculares en especies como Streblonema penetrale, Asteronema breviarticulatum, Ectocarpus siliculosus y Bachelotia antillarum, tanto en la época de lluvias como en la de secas. Sargassum howellii,
S. liebmanii y Padina crispata presentaron oogonios y anteridios. En contraste, en Chlorophyta sólo el 21.43\% de las especies presentó algún estado reproductivo, lo que puede deberse a que las especies se encontraban en estadios juveniles y a que las fases sexuales de estos organismos tienen un periodo corto de vida (Santelices, 1977, Littler et al., 1983).

Especies epifitas. El epifitismo puede ser considerado como una importante estrategia ecológica, con el fin de obtener un sustrato en condiciones óptimas de luz y 
protección adecuada para el establecimiento y desarrollo de estas especies. En adición, el epifitismo es una variable importante en el estudio de la estructura de las comunidades marinas bentónicas, al realizar un detallado análisis de las epifitas, se incrementa la riqueza específica de algas marinas registrada para determinada región (Montañés et al., 2003, Meneses y Faria, 2008).
En el presente estudio se observó la relevancia de esta variable, en las figuras 6 y 7, se muestra que durante todo el periodo de estudio se presentaron especies epífitas. El mayor número de epífitas se encontró en la estación climática de secas con 33 especies, observándose en este hábitat un incremento en el número de taxa de Cyanophyta y de las familias Ectocarpaceae y Ceramiaceae las cuales

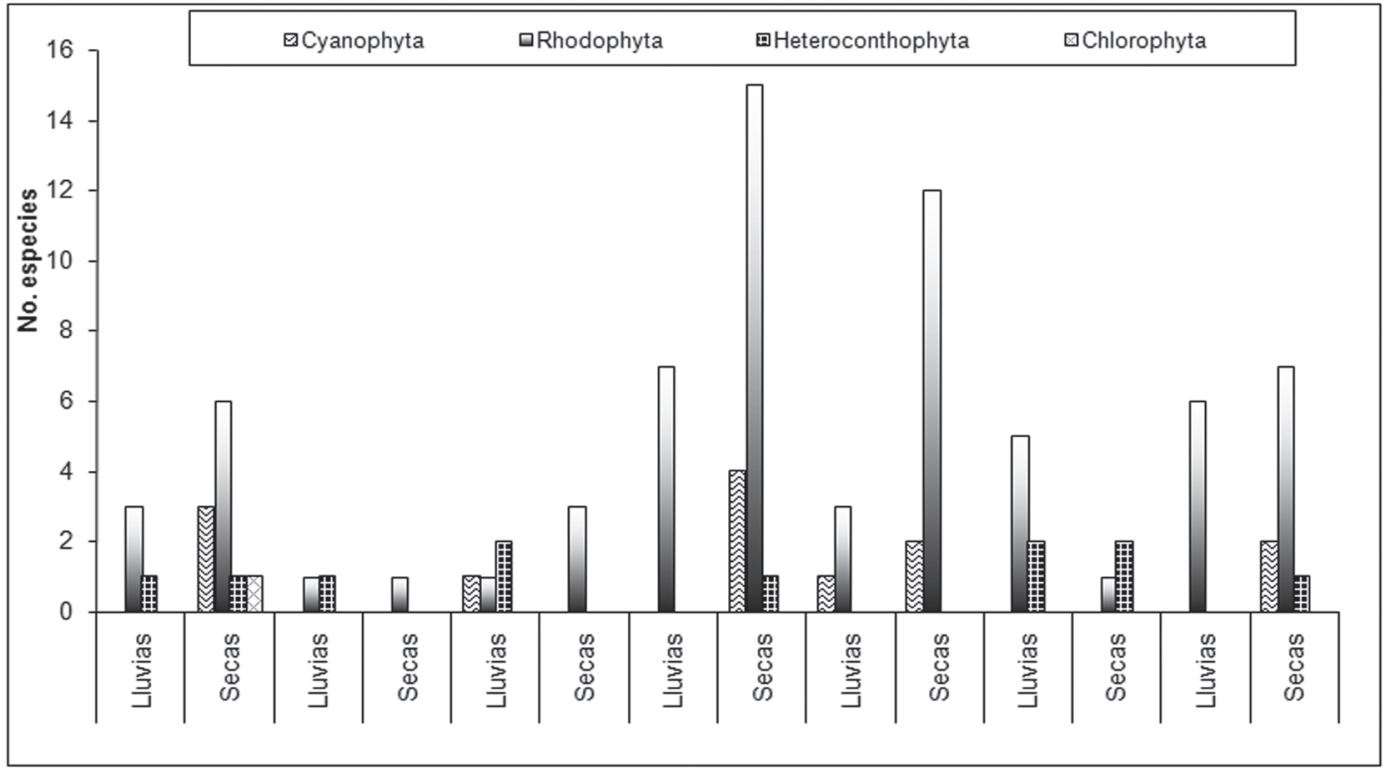

Figura 6. Número de especies epífitas por localidad y estación climática.

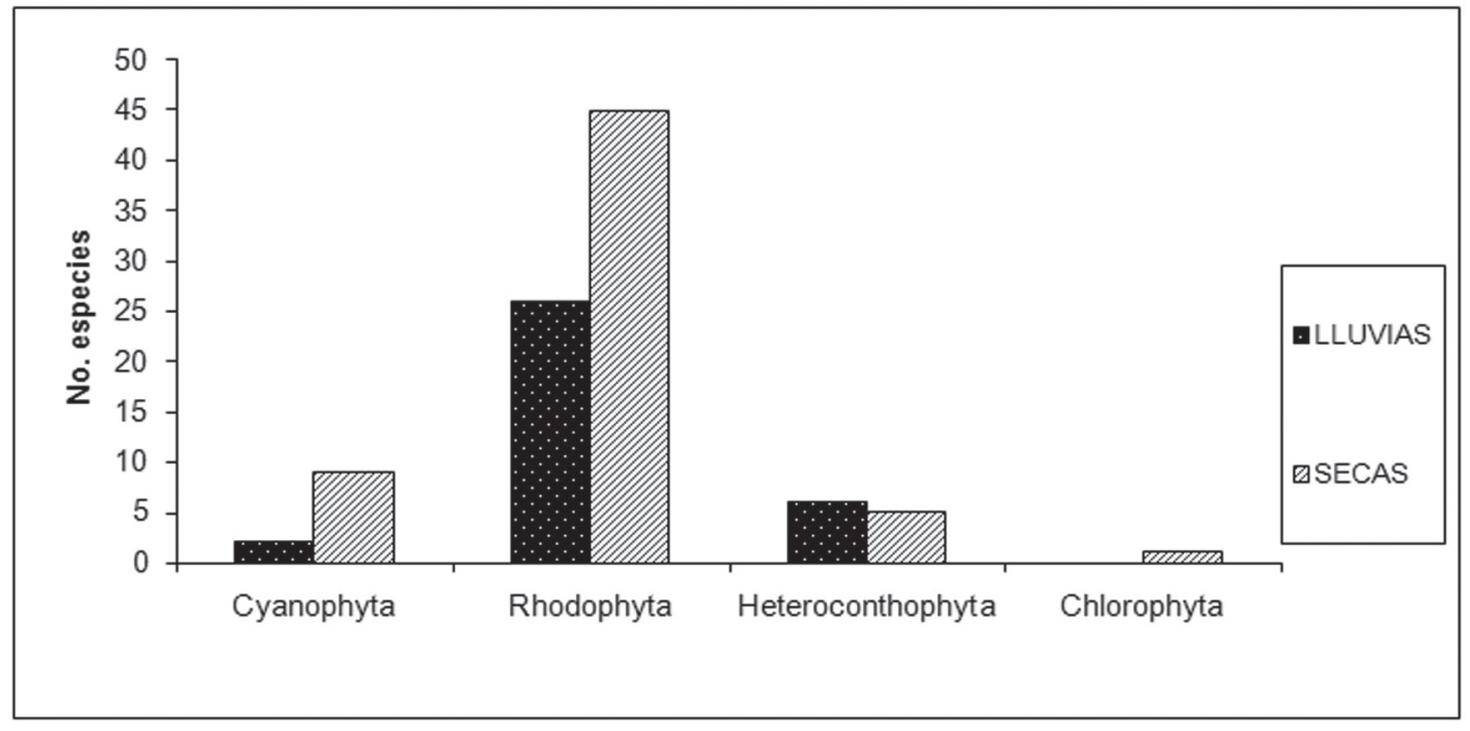

Figura 7. Total de especies epifitas por estación climática. 
epifitan a sus hospederos hasta casi $90 \%$ de su superficie, cuando éstos se encuentran en decadencia en esta estación climática, a diferencia de la época de lluvias donde las poblaciones de macroalgas bentónicas se encuentran en florecimiento (Meneses y Faria, 2008; Mateo-Cid y Mendoza-González, 1991). En conjunto se localizaron 49 especies epífitas, de las cuales 40 son epífitas estrictas; estas últimas se encuentran señaladas en el Apéndice 1. Del total de epífitas, 10 especies corresponden a Cyanophyta, 33 a Rhodophyta, 5 a Heteroconthophyta y 1 a Chlorophyta. De acuerdo con Montañés et al. (2003), los talos laminares y flabelados, como los del género Padina, constituyen un sustrato idóneo para el establecimiento de numerosas epífitas. Por otro lado, la mayoría de las epífitas se encontraron creciendo frecuentemente sobre algas perennes como Amphiroa beauvoisii y A. misakiensis, además de Chaetomorpha anteninna, Sargassum howellii y S. liebmanii; en este contexto se ha sugerido que la longevidad del huésped debe ser lo suficientemente larga para permitir al epífito completar su ciclo vital, lo que podría ser una razón probable de la ausencia de epífitas en las algas anuales y efímeras (Santelices, 1977). Asimismo, el elevado número de epífitas encontradas en este estudio se ha observado en otros sitios cercanos, como Manzanillo, Puerto Vallarta y Oaxaca (Mateo Cid y Mendoza González, 1991, 2001, Mendoza González y Mateo Cid, 1992).

El análisis y la integración de los estudios previos y los resultados obtenidos en el presente estudio permiten visualizar la importancia de realizar inventarios ficoflorísticos en el litoral mexicano. Además, este tipo de investigaciones permite conocer las regiones aún no exploradas. Para obtener un conocimiento más preciso de los recursos algales de nuestro país,son necesarios estudios como el presente.

\section{Agradecimientos}

Al Instituto Politécnico Nacional por facilitar las instalaciones y equipo necesario para el desarrollo de este estudio. La primera autora agradece la beca otorgada por la Comisión de Operación y Fomento de Actividades Académicas (COFAA). A los revisores anónimos que con sus comentarios y sugerencias mejoraron notablemente este manuscrito.

\section{Literatura citada}

Abbott, I. A. y G. J. Hollenberg. 1976. Marine algae of California.
Stanford Univerty Press, California. 789 p.

Águila-Ramírez, N., A. Gaspar Figueroa, I. Enciso Padilla y M. R. Mora Navarro.1998. Algas marinas de la costa sur de Jalisco. Boletín del Instituto de Botánica (Universidad de Guadalajara) 5:507-514.

Anagnostidis, K. y J. Komárek. 1988. Modern approach to the classification system of Cyanophytes. 3. Oscillatoriales. Archv fur Hydrobiologie, Supplement 80:327-472.

Ardito, S. y S. Gómez. 2005. Patrón fenológico de una población de Gelidium serrulatum J. Agardh (Rhodophyta, Gelidiales) en la localidad de Taguao, Estado Vargas, Venezuela. Acta Botanica Venezuelica 28:101-111.

Báez, J. C., R. Real, J.M. Vargas y A. Flores-Moya. 2004. Revisión critica de los estudios sobre biogeografía de macroalgas marinas del Mediterráneo. Acta Botanica Malacitana 29:5-11

Chávez-Barrera, M. L. 1972. Estudio de la flora marina de la bahía de Zihuatanejo y lugares adyacentes. Memorias del IV Congreso Nacional de Oceanografía. 17-19 noviembre de 1969. México, D. F. 265-271.

Chávez-Barrera, M. L. 1980. Distribución del género Padina en las costas de México. Anales Escuela Nacional de Ciencias Biológicas, México 23:45-51.

Cheney, D.P. 1977. A new improved ratio for comparing seaweed floras. Journal of Phycology 13(supplement):1-13.

Cho, T. O., Sung Min Boo, M. H. Hommersand, C. A. Maggs, L. McIvor y S. Fredericq. 2008. Gayliella gen. nov. in the Tribe Ceramieaea (Ceramiaceae, Rhodophyta) based on molecular and morphological evidence. Journal of Phycology 44:721738 .

Dawson, E. Y. 1949. Resultados preliminares de un reconocimiento de las algas marinas de la costa pacífica de México. Revista de la Sociedad Mexicana de Historia Natural 9:215-255.

Dawson, E. Y. 1951. A further study of upwelling and associated vegetation along Pacific Baja California, México. Journal of Marine Research 10:39-58.

Dawson, E. Y. 1953a. Resumen de las Investigaciones recientes sobre algas marinas de la costa pacífica de México, con una sinopsis de la literatura, sinonimia y distribución de las especies descritas. Revista de la Sociedad Mexicana de Historia Natural 13:97-197. 1 tabla.

Dawson, E. Y. 1953b. Marine red algae of Pacific Mexico I. Bangiales to Corallinoideae. Allan Hancock Pacific Expeditions 17:1-239.

Dawson, E. Y. 1954. Marine red algae of Pacific Mexico II. Cryptonemiales. Allan Hancock Pacific Expeditions 17:241397.

Dawson, E. Y. 1960. Marine red algae of Pacific Mexico III. Cryptonemiales. Corallinaceae, subfamily Melobesioideae. Pacific Naturalist 28:1-125.

Dawson, E.Y. 1961. A guide to the literature and distribution of Pacific benthic algae from Alaska to the Galapagos islands. Pacific Science 15:370-461 
Dawson, E. Y. 1963a. Marine red algae of Pacific México Part 6. Rhodymeniales. Nova Hedwigia 5:437-476.

Dawson, E. Y. 1963b. Marine red algae of Pacific Mexico. Part 8.Ceramiales: Dasyaceae, Rhodomelaceae. Nova Hedwigia 6:437-476

Dreckmann, K. M., F. F. Pedroche y A. Sentíes-Granados. 1990. Lista florística de las algas marinas bentónicas de la costa norte de Michoacán, México. Boletín de la Sociedad Botánica de México 50:19-42.

Dreckmann, K. M., A. Sentíes G., F. F. Pedroche y M. Callejas. 2006. Diagnostico florístico de la ficología marina bentónica en Chiapas. Hidrobiológica 16:147-158.

Enciso-Padilla, I, N. Aguilar Ramírez, M. Mora Navarro y A. Gaspar Figueroa. 1995.

Macroalgas de la zona intermareal de Melaque, Jalisco, México. Boletín del Instituto de Botanica [Universidad de Guadalajara] 3:41-51

Feldmann, J. 1937. Recherches sur la vegetation marine de la Mediterranée. La Cote des Alberes. Revue Algologique 10:1339.

Galicia-García, C. 1998. Estudio florístico de las algas marinas bentónicas de la costa sur de Jalisco, México. Tesis, Escuela Nacional de Ciencias Biológicas, IPN, México, D.F. 138 p.

García, E. 1980. Modificaciones al sistema de clasificación climática de Köppen (Para adaptarlo a las condiciones de la República Mexicana). Instituto de Geografía, UNAM, México, D. F. 246 p.

Guiry, M.D y W.D. Guiry. 2008. AlgaeBase version 4.2. Worldwide electronic publication, National University of Ireland, Galway. http://www.algaebase.org; 30.IX.2009

Hollenberg, G. J. 1961. Marine red algae of Pacific Mexico. The genus Polysiphonia. Pacific Naturalist 2:345-375.

León-Álvarez, D., E. Serviere-Zaragoza y J. González-González. 1997. Description of the tetrasporangial crustose and gametangial erect phases of Ahnfeltiopsis gigartinoides (J. Agardh) Silva et DeCew (Rhodophyta, Phyllophoraceae) in Bahía de Banderas, México. Botanica Marina 40:397-404.

Littler, M.M., D. S. Littler, y P. R. Taylor. 1983. Evolutionary strategies in a tropical barrier reef system: functional-form groups of marine macroalgae. Journal of Phycology 19:229237.

Magalhães Lucio, A. y J.M de Castro Nunes. 2002. Aportación al conocimiento fenológico de las rodofíceas marinas de la playa del Guarajuba (Camaçari, Bahía) Brasil. Botanica Complutensis 26:17-34.

Mateo-Cid, L E. y A C. Mendoza-González. 1991. Algas marinas bénticas de la costa del estado de Colima, México. Acta Botanica Mexicana 13:9-30.

Mateo-Cid, L. E., y A. C. Mendoza-González. 1992. Algas marinas bentónicas de la costa sur de Nayarit, México. Acta Botanica. Mexicana 20:13-28.

Mateo-Cid, L.E. y A.C. Mendoza-González. 2002 [2001]. Algas marinas bentónicas de la costa de Oaxaca, México. Anales de la Escuela Nacional de Ciencias Biológicas, México 47:1123.

Mendoza-González, A. C. y L. E. Mateo-Cid. 1992. Estudio preliminar de las algas marinas bentónicas de la costa de Jalisco, México. Anales de la Escuela Nacional de Ciencias Biológicas, México 37:9-25.

Mendoza-González, A.C. y L.E. Mateo-Cid. 1998. Avance de un estudio sobre las macroalgas marinas de Guerrero y Oaxaca. Ciencia y Mar 4:15-29.

Menezes de Széchy, M.T. y A. D. Faria de Sá. 2008. Variacão sazonal do epifitismo por macroalgas em una populacão de Sargassum vulgare C. Agardh (Phaeophyceae, Fucales) da Bahia da Ilha Grande, Rio de Janeiro. Oecologia Brasileira 12:299-314.

Montañés, M. A., J. Reyes y M. Sansón. 2003. La comunidad de epífitos de Zonaria tournefortii en el norte de Tenerife (islas Canarias); análisis florístico y comentarios sobre su epifauna. Vieraea 31:121-132.

Morales-Ayala, S. y M.A. Viera-Rodríguez. 1989. Distribución de los epífitos en Cystoseira tamariscifolia (Hudson) Papenfuss (Fucales, Phaeophyta) en Punta de Gáldar (Gran Canaria, islas Canarias). Anales del Jardín Botánico de Madrid 46:107-113.

Norris, J.N. 2010. Marine algae of the northern Gulf of California: Chlorophyta and Phaeophyceae. Smithsonian Contributions to Botany 94:1-276.

Norris, J.N. y H. W. Johansen. 1981. Articulated coralline algae of the Gulf of California, México. I. Amphiroa Lamouroux. Smithsonian Contributions to the Marine Sciences 9:1-29.

Pedroche, F. F. y J. González-González. 1981. Lista florística preliminar de las algas marinas de la región sur de la costa de Jalisco, México. Phycologia Latino-Americana. 1:60-71.

Pedroche, F. F., P. C. Silva, L. E. Aguilar-Rosas, K. M. Dreckmann y R. Aguilar-Rosas. 2005. Catálogo de las algas marinas bentónicas del Pacífico de México. I. Chlorophycota. Universidad Autónoma Metropolitana/ Universidad Autónoma de Baja California/ University of California, México, D.F. 135 p.

Pedroche, F. F., P. C. Silva, L. E. Aguilar-Rosas, K. M. Dreckmann y R. Aguilar-Rosas. 2008. Catálogo de las algas marinas bentónicas del Pacífico de México. II. Phaeophycota. Universidad Autónoma Metropolitana/ Universidad Autónoma de Baja California/ University of California, México, D.F. 146 p.

Rodríguez, D., N. López y J. González-González. 2008. Gelidiales (Rhodophyta) en las costas del Pacífico mexicano con énfasis en las especies tropicales. In Monografías ficológicas, A. Sentíes G. y K. Dreckmann (eds.) Universidad Autónoma Metropolitana / Universidad Autónoma de Baja California, México, D.F. p. 27-74.

Santelices, B. 1977. Ecología de las algas marinas bentónicas. 
Universidad Católica de Chile, Santiago. 384 p.

Serviere-Zaragoza, E., J. González-González y D. RodríguezVargas. 1993. Ficoflora de la región de bahía Banderas, Jalisco-Nayarit. In Biodiversidad marina y costera de México, S. I. Salazar Vallejo y N. E. González (eds.). Comisión Nacional para el Conocimiento y Uso de la Biodiversidad y Centro de Investigaciones de Quintana Roo, México. p. 475485.

Serviere-Zaragoza, E., S. Castillo Arguero y J. GonzálezGonzález. 1998. Descripción ficológica de los ambientes de la región de bahía de Banderas, Nayarit-Jalisco,
México. Boletín del Instituto de Botánica [Universidad de Guadalajara]. 5:157-180.

Taylor, W. R. 1945. Pacific marine algae of the Allan Hancock Expeditions to the Galapagos Islands. Allan Hancock Pacific Expeditions 12:1-528.

Taylor, W.R. 1960. Marine algae of the eastern tropical and subtropical coast of the Americas. University of Michigan Press, Ann Arbor. 870 p.

Wynne, M. J. 2005. A checklist of benthic marine algae of the tropical and subtropical western Atlantic: second revision. Nova Hedwigia Beiheft 129:152 p. 


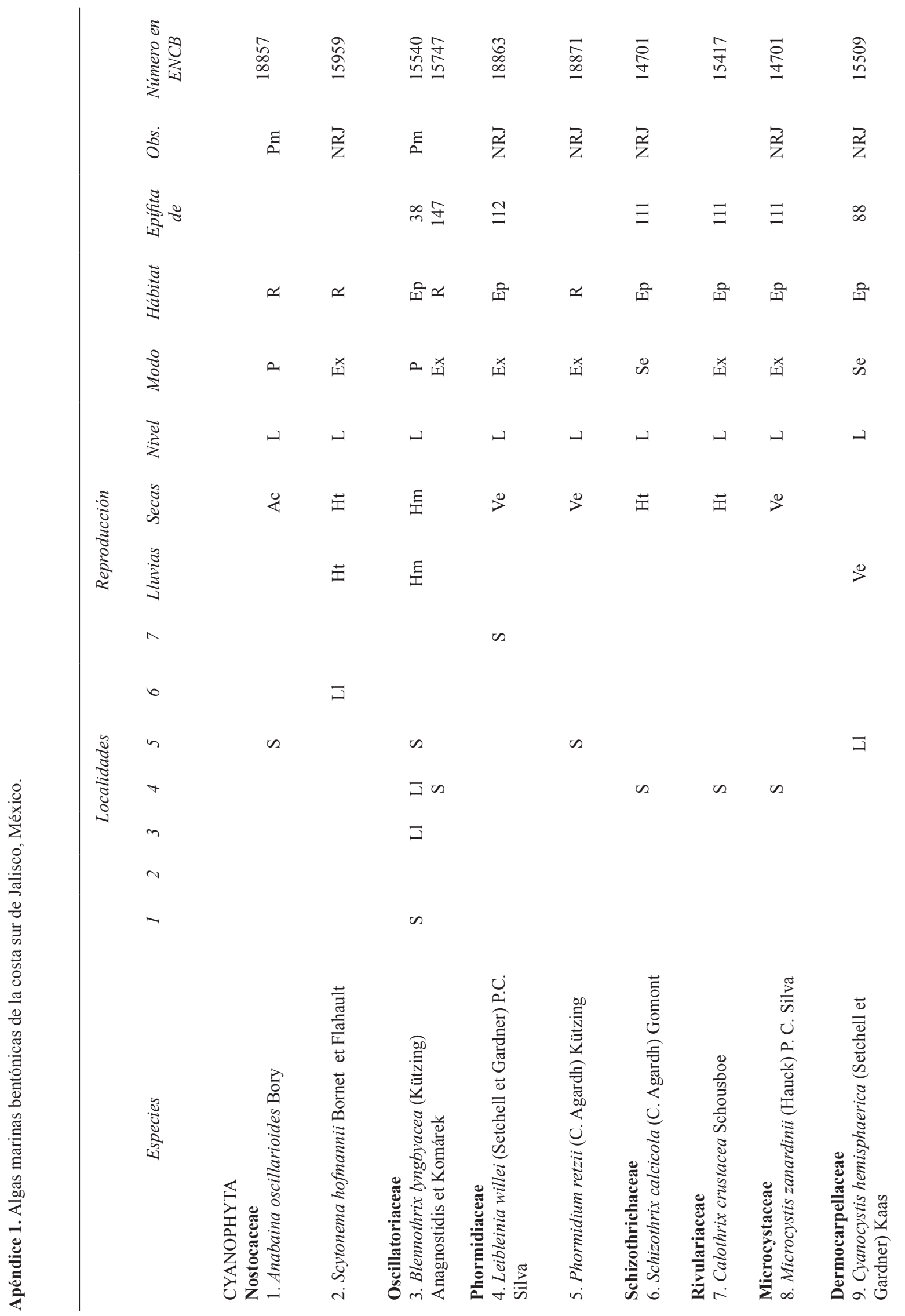




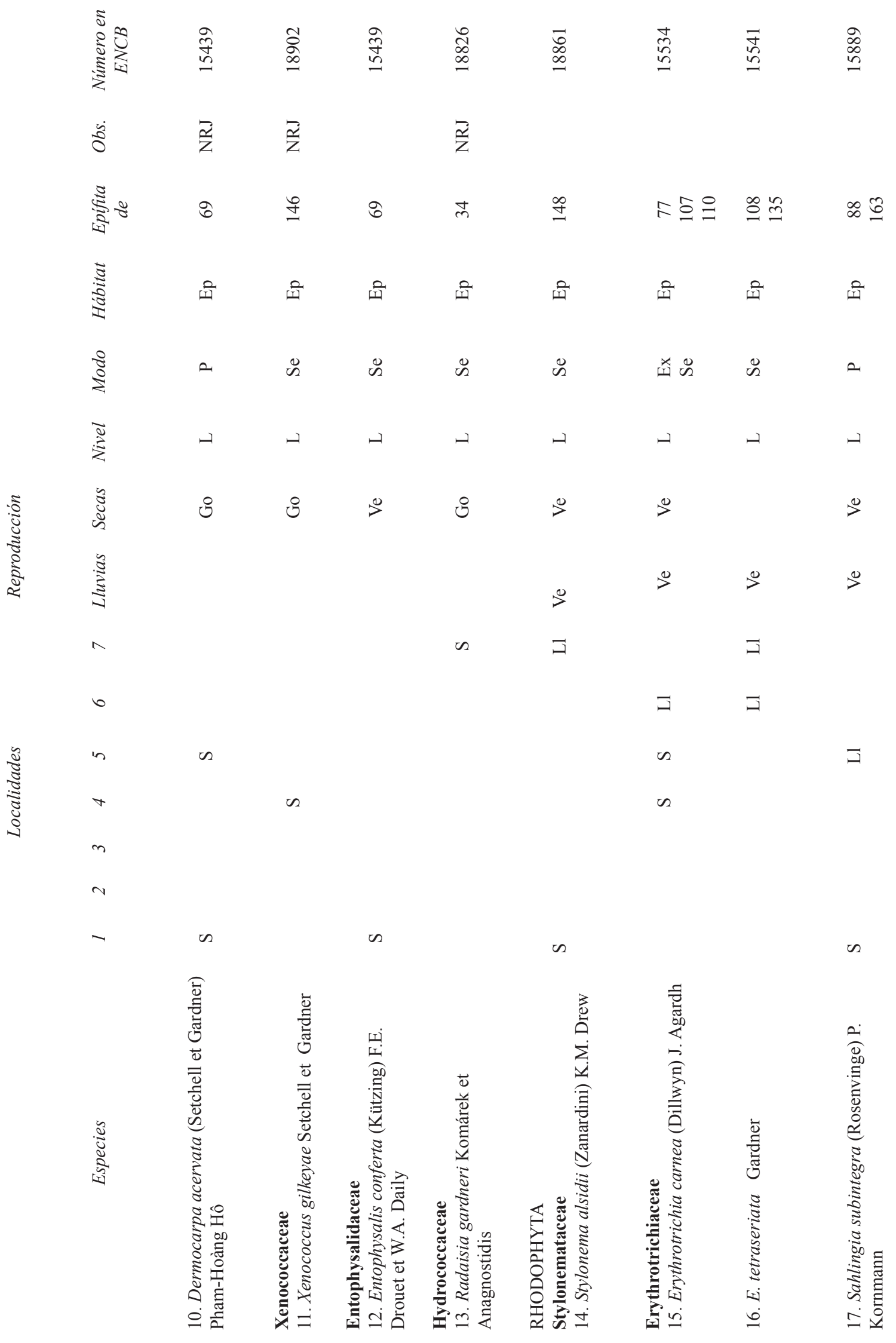


Revista Mexicana de Biodiversidad 82: 19-49, 2011

33

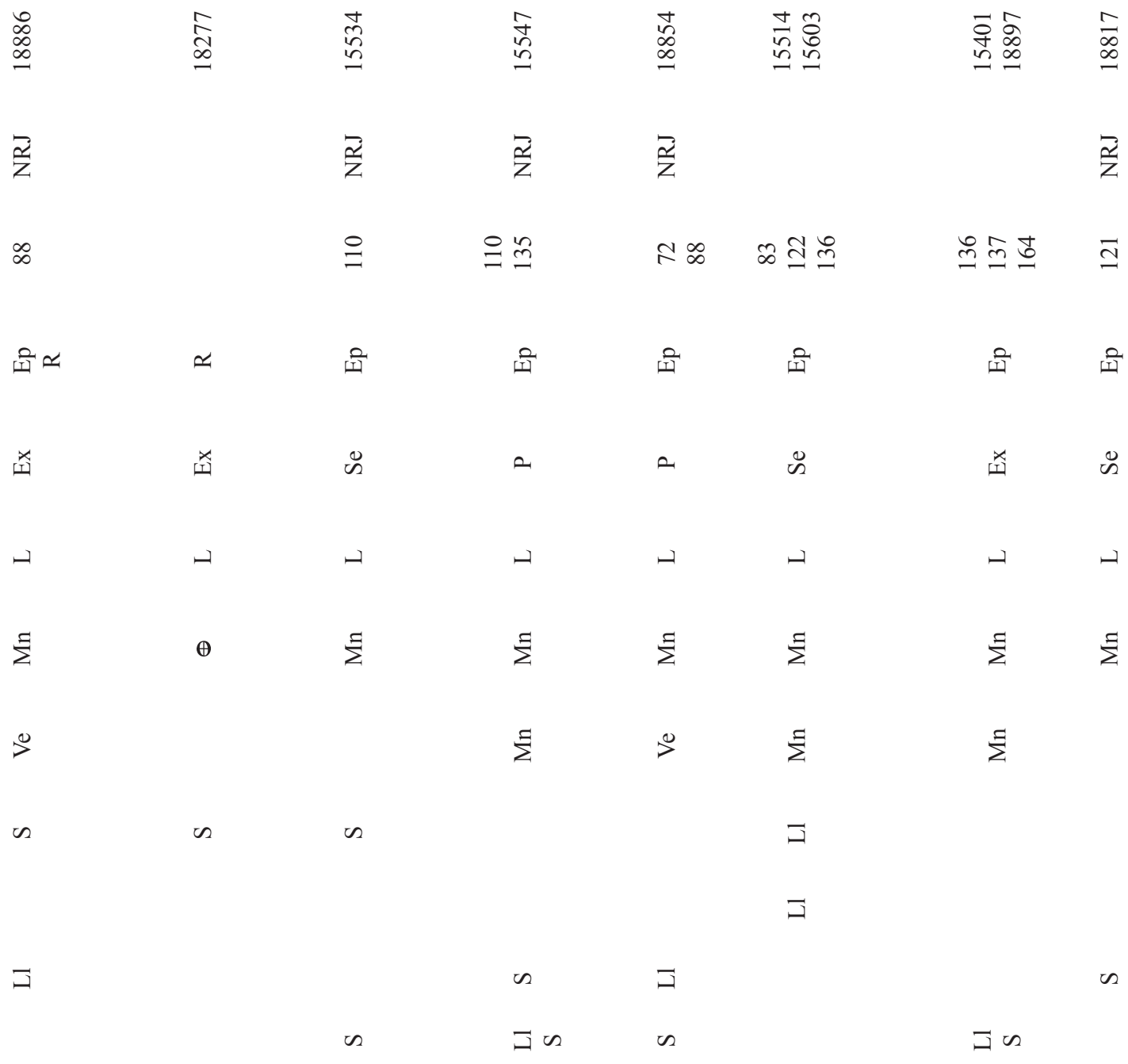

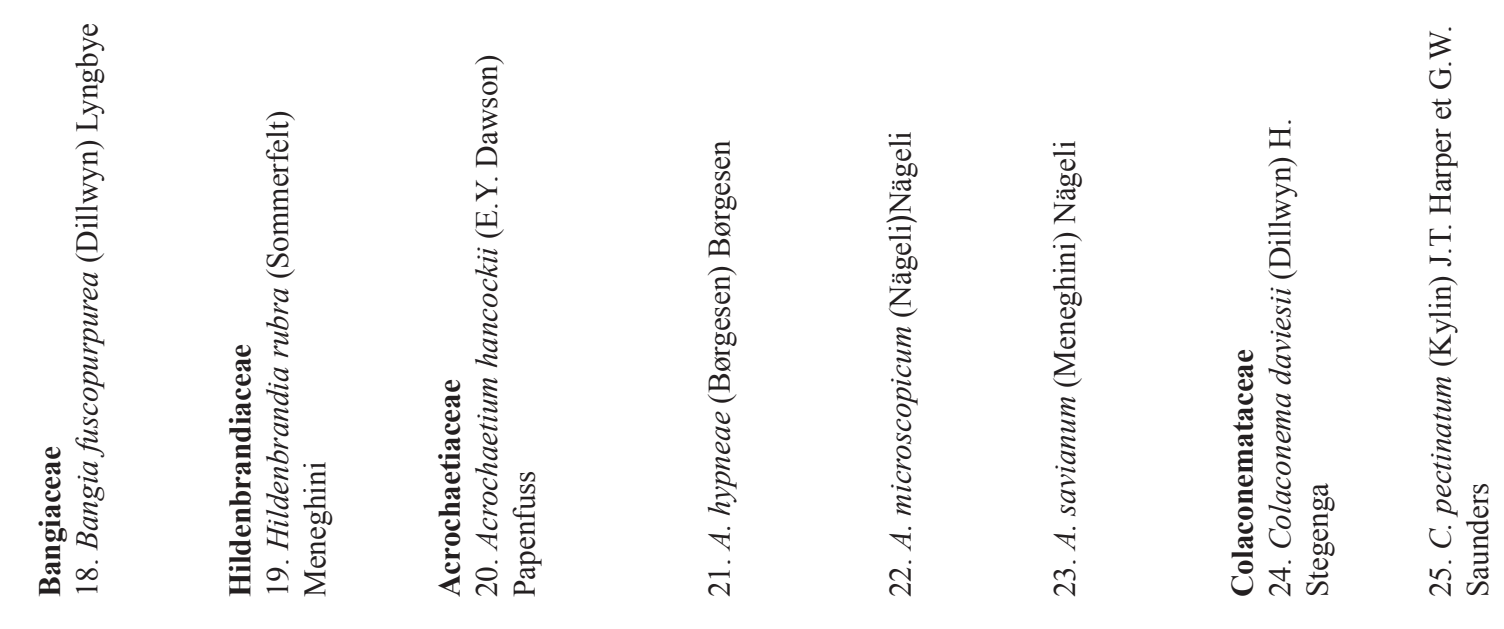




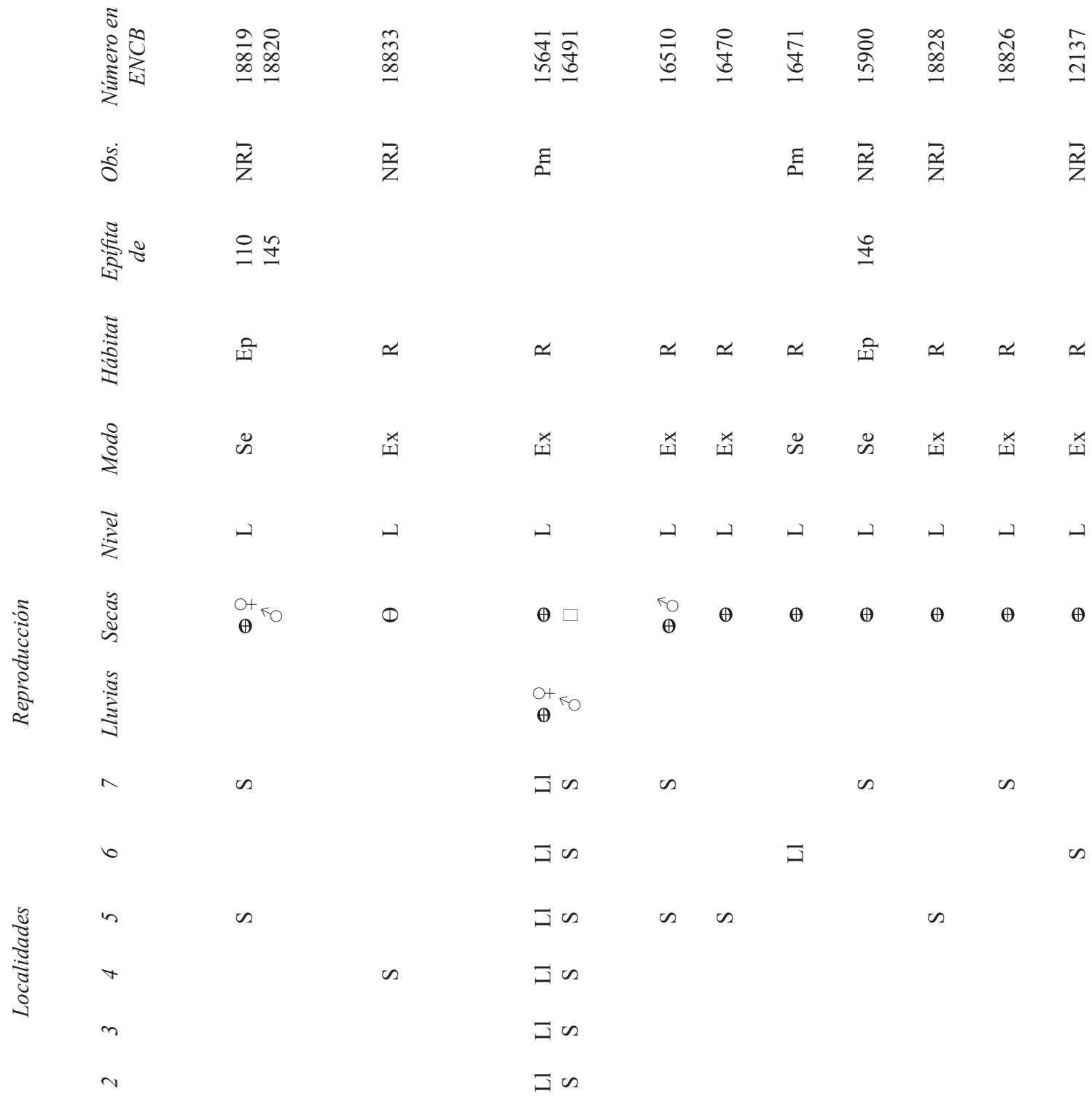




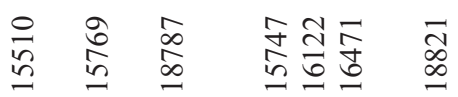

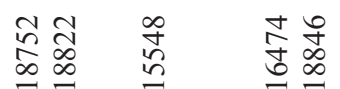

$$
2
$$

$\Xi$

z

$\Xi \widetilde{a}$

$\infty$

守㐫

$\infty \underset{\Xi}{\infty}$

$\underline{ }$

위

$\simeq \simeq$ 외

N

뇌

$\simeq$

$\propto$

되 워

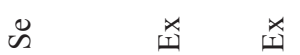

$\ddot{\infty}$

$\ddot{\infty}$

盾

近

圣 ๗

$\oplus \quad \oplus$

$\kappa_{0}$
$\theta$

ro

ro
$\oplus$

$\theta$

$\oplus$

$\begin{array}{lll}\oplus & \oplus \\ & \oplus & \oplus\end{array}$

$\oplus$

5
$\theta$

$\theta$

$\oplus$

$\oplus$

$\infty$ is

$$
\text { a }
$$

$\infty$

ヨル

๘

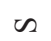

n

2

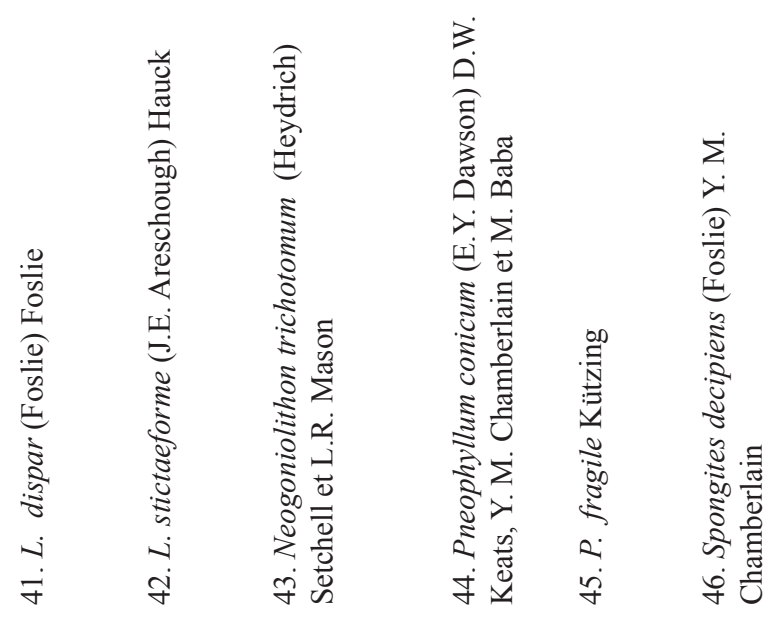




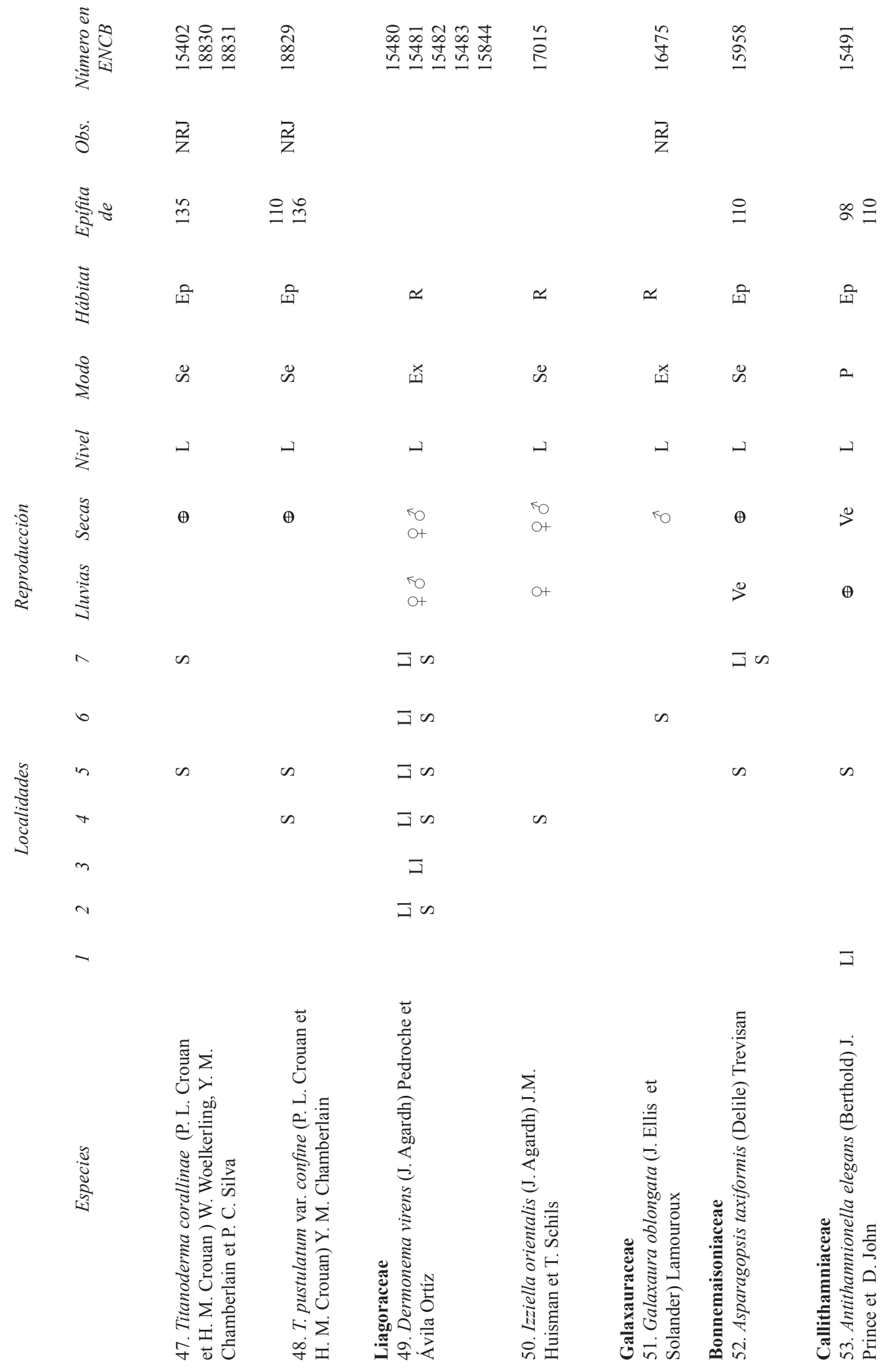




\begin{tabular}{|c|c|c|c|c|c|c|c|c|}
\hline $\begin{array}{l}n \\
\infty \\
\infty\end{array}$ & 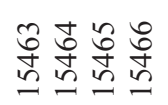 & 疍 & $\begin{array}{l}\stackrel{+}{\infty} \\
\infty \\
\infty\end{array}$ & $\begin{array}{l}\hat{\infty} \\
\infty \\
\infty\end{array}$ & $\begin{array}{l}\text { む゙ } \\
\stackrel{\infty}{\infty}\end{array}$ & 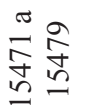 & 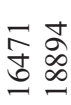 & $\begin{array}{l}0 \\
\text { : } \\
n \\
n\end{array}$ \\
\hline & & & & & & & $\vec{z}$ & \\
\hline 8 & $\underset{n}{\infty}$ & & & $\stackrel{\mathscr{P}}{ \pm}$ & $\bar{\sigma}$ & $\stackrel{\infty}{\sim}$ & $\underset{m}{\infty}$ & $\stackrel{\varrho}{=}$ \\
\hline & $\simeq$ ્ㅚ & $\simeq$ & N & ્ㅚ & $\begin{array}{l}\text { 워 } \\
\text { ئ }\end{array}$ & 노 워 & भิد & 외 \\
\hline 0 & $\stackrel{n}{ }$ & 盾 & $\ddot{n}$ & $\ddot{n}$ & 狅 & $\ddot{n}$ & $\ddot{n}$ & $\ddot{~}$ \\
\hline & لـ & ــ & دـ & دــ & ـــ & ــ & 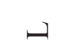 & دـ \\
\hline & $\begin{array}{c}\oplus \\
\text { O+ }\end{array}$ & & $\Theta$ & & ro & $\mathrm{O}_{\theta}^{+} \mathrm{r}$ & $\begin{array}{l}\mathrm{r}_{0} \\
\boldsymbol{\theta}\end{array}$ & $\begin{array}{c}\text { O+ } \\
\theta\end{array}$ \\
\hline
\end{tabular}

$\Theta$

$\Theta$

$\Theta$

$\stackrel{\text { O+ }}{\oplus}$ זO

n

is

n

コ

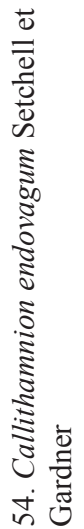

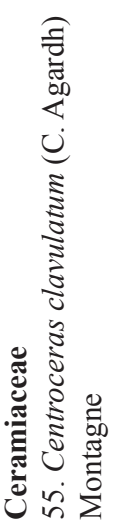




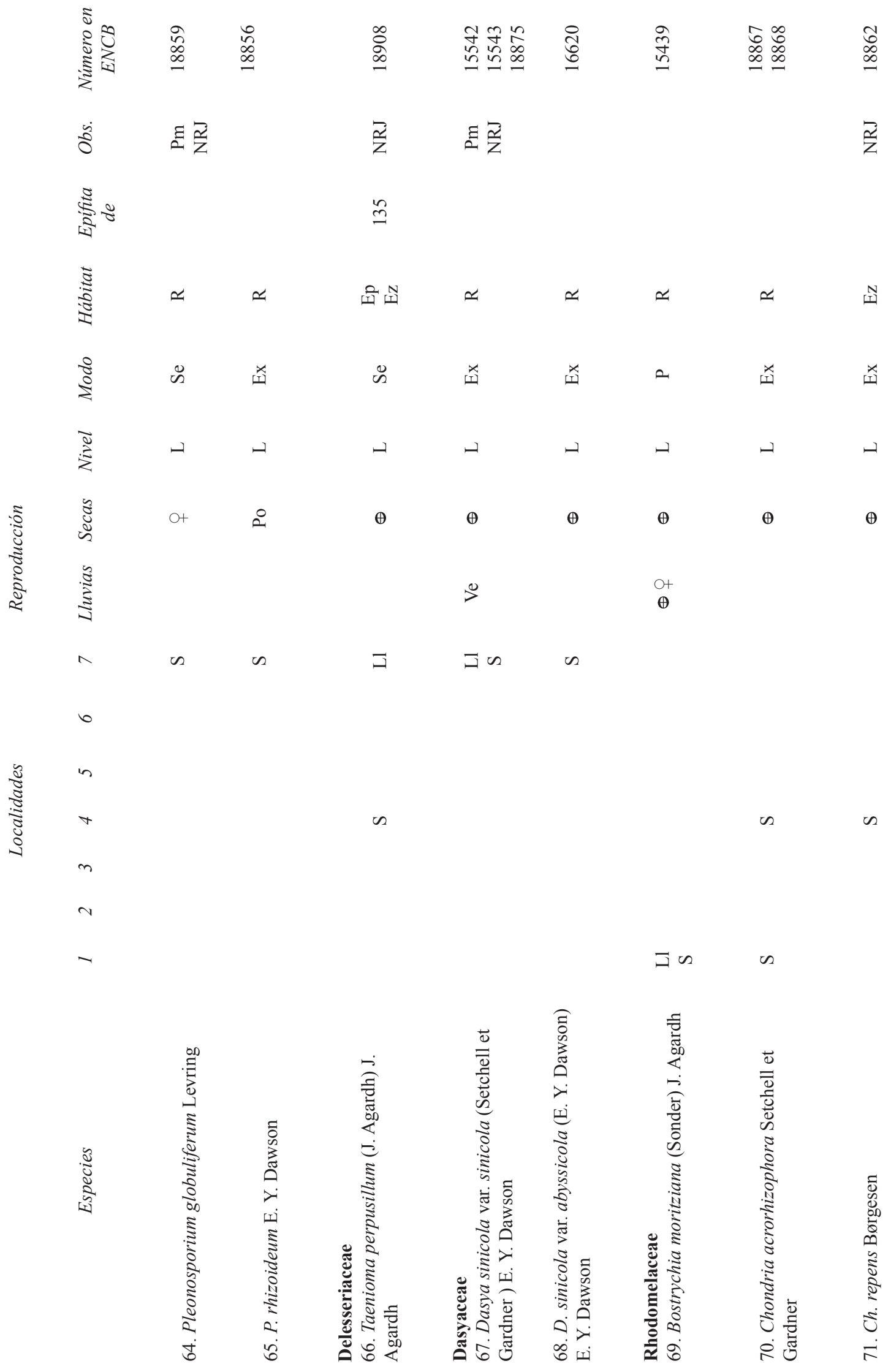




\begin{tabular}{|c|c|c|c|c|c|c|c|c|c|c|}
\hline $\begin{array}{l}\infty \\
\infty \\
\infty \\
\infty\end{array}$ & $\begin{array}{l}0 \\
n \\
n \\
n\end{array}$ & \begin{tabular}{l}
0 \\
\multirow{2}{n}{} \\
\end{tabular} & 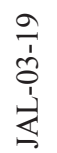 & $\begin{array}{l}\text { 导㠻 } \\
\text { 点 }\end{array}$ & 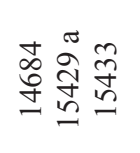 & $\begin{array}{l}\infty \\
\infty \\
\infty \\
\infty\end{array}$ & $\begin{array}{l}\text { Oे } \\
\stackrel{2}{n}\end{array}$ & $\begin{array}{l}\infty \\
\stackrel{0}{0} \\
\stackrel{2}{n}\end{array}$ & $\begin{array}{l}\stackrel{2}{\infty} \\
\infty \\
\infty\end{array}$ & $\begin{array}{l}\stackrel{+}{+} \\
\stackrel{f}{n}\end{array}$ \\
\hline & $\vec{z}$ & $\vec{z}$ & $\vec{z}$ & & & $\vec{z}$ & $\vec{z}$ & & $\vec{z}$ & $\Xi$ \\
\hline 8 & $\stackrel{ }{=}$ & & & & & $\infty$ & & $\cong$ & & \\
\hline 표 & 今િ & $\stackrel{N}{I}$ & $\simeq$ & $\simeq$ & $\simeq$ & 워 & $\simeq$ & 외 & $\simeq$ & $\simeq$ \\
\hline 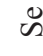 & $\ddot{n}$ & $\ddot{n}$ & 差 & 质 & 左 & $\ddot{n}$ & 兲 & $\ddot{n}$ & 后 & 差 \\
\hline ــ & دـ & \lrcorner & \lrcorner & ــ & \lrcorner & 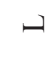 & $ـ$ & $\dashv$ & - & لـ \\
\hline$\oplus$ & ${ }_{\boldsymbol{\theta}}^{\mathrm{NO}} \mathrm{O+}$ & $\Theta$ & O+ & & $\stackrel{\text { O+ }}{\oplus}$ & & 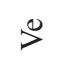 & & $\oplus$ & $\Theta$ \\
\hline & & & & O+ & $\begin{array}{c}\mathrm{O}+ \\
\Theta\end{array}$ & O+ & & ro & & \\
\hline & & & & & & コ & $\sim$ & & & \\
\hline & $\infty$ & & is & & コ & & & & & is \\
\hline & & is & & & $n$ & & & コ & & \\
\hline & & & & コ & コ & & & & & \\
\hline & & & & & コ & & & & & \\
\hline & & & & コ & コ & & & & os & \\
\hline
\end{tabular}
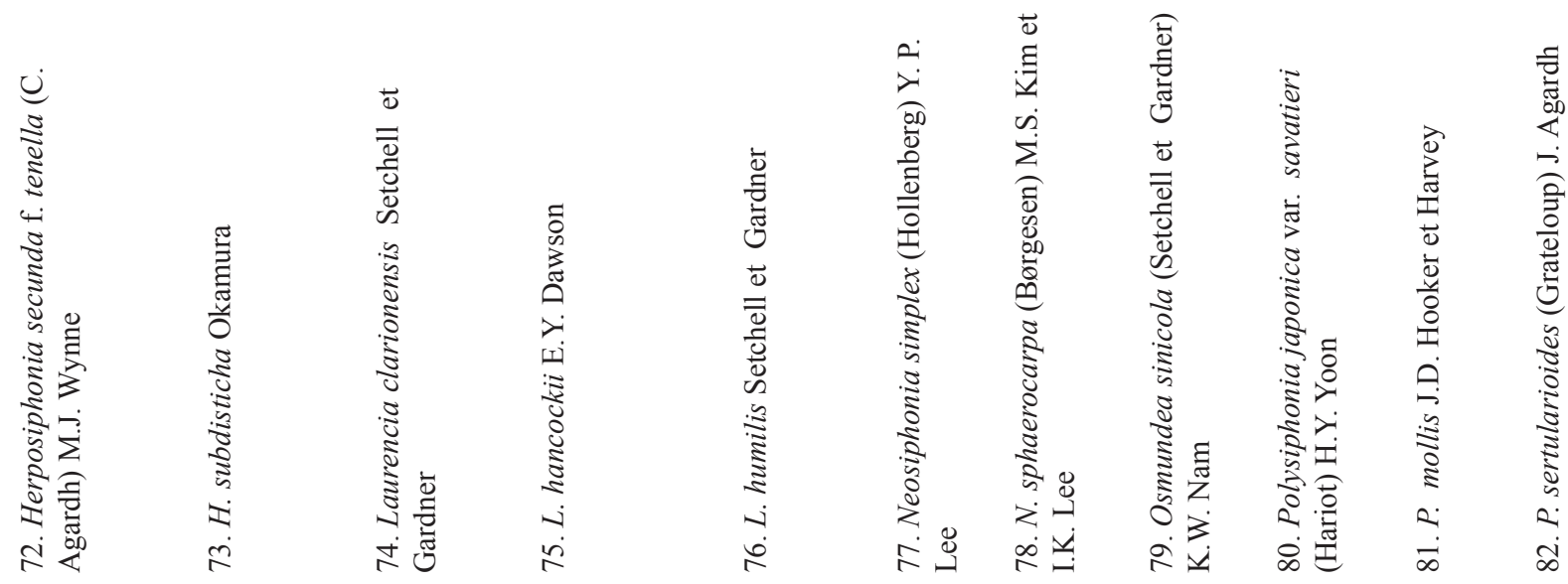


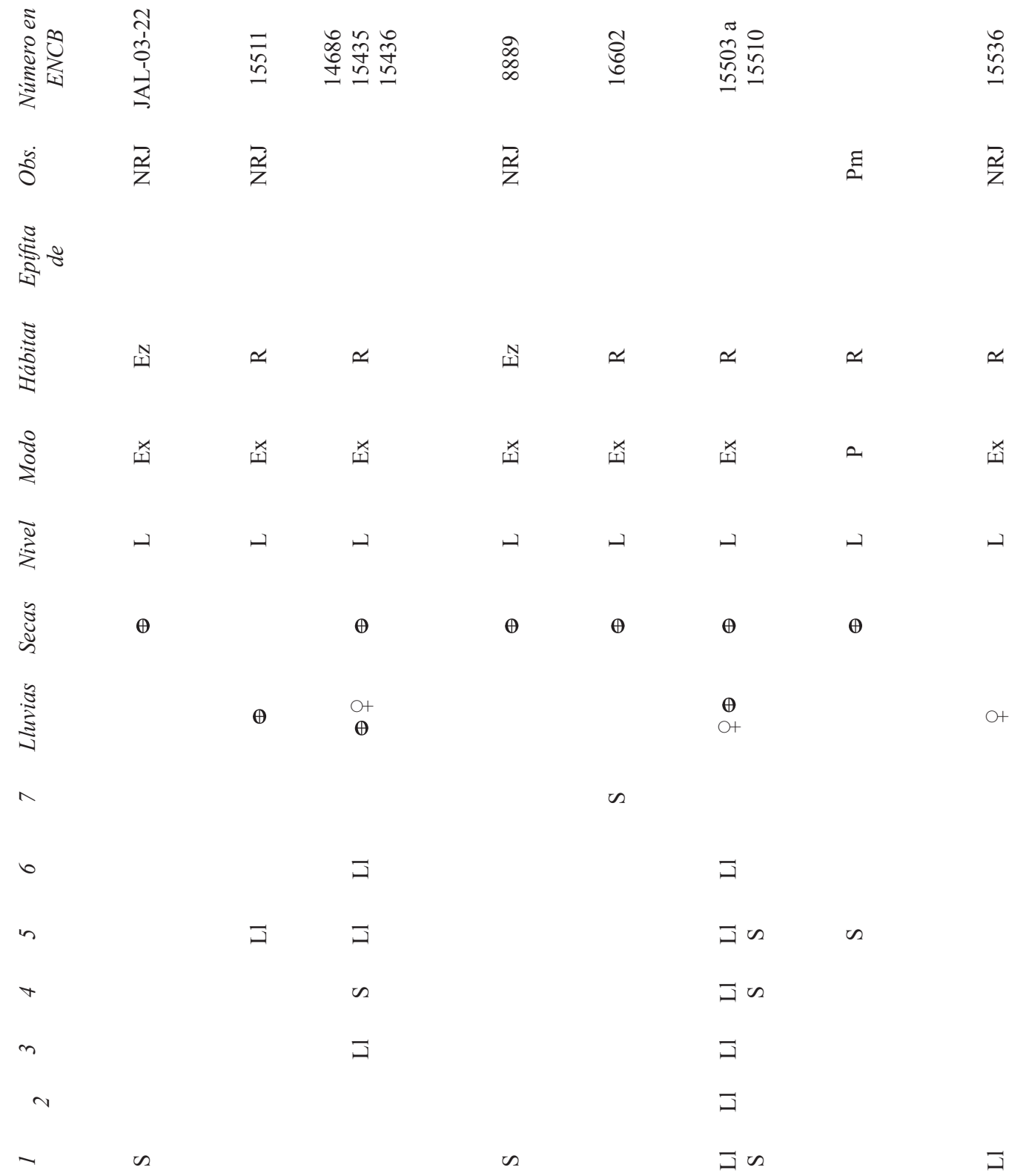

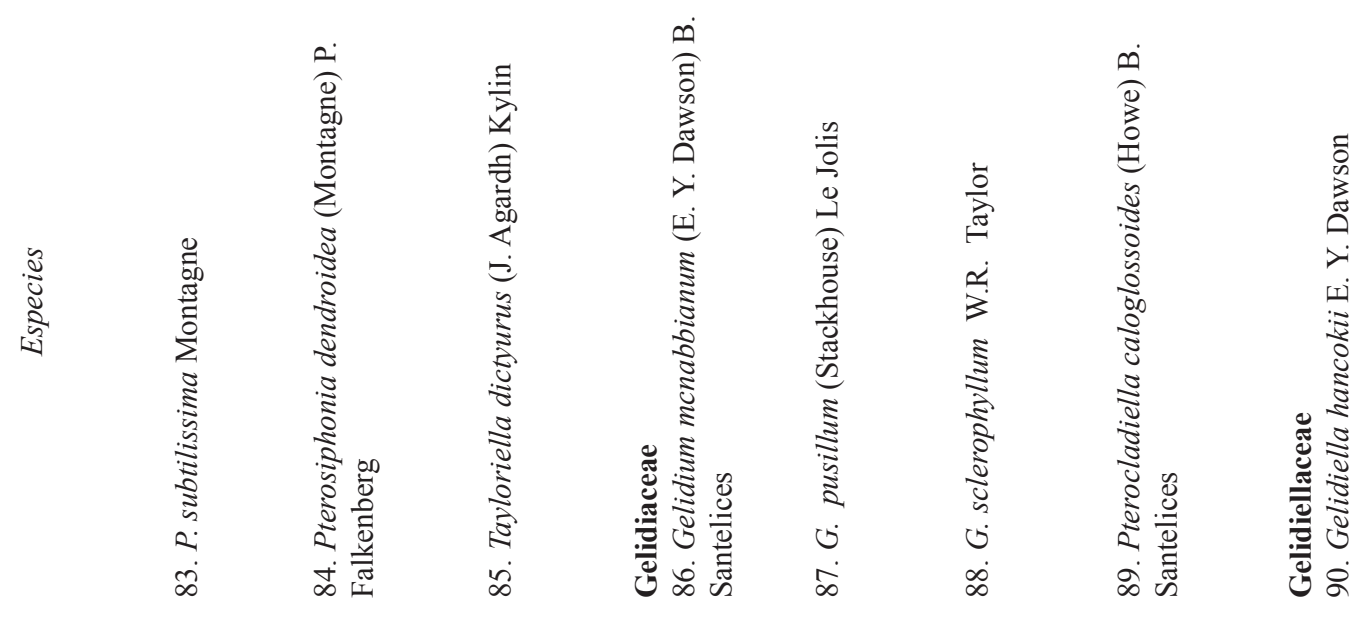




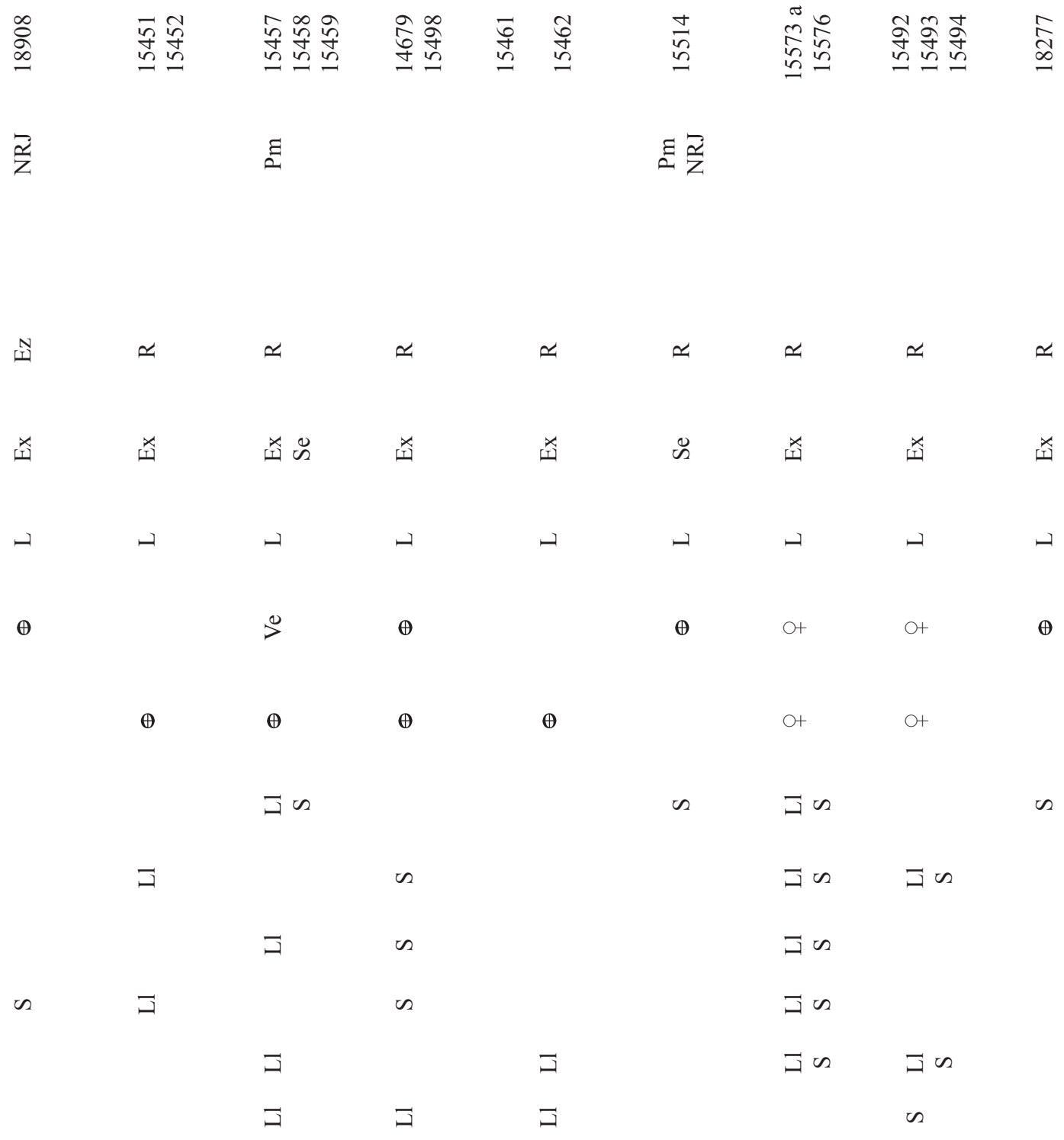

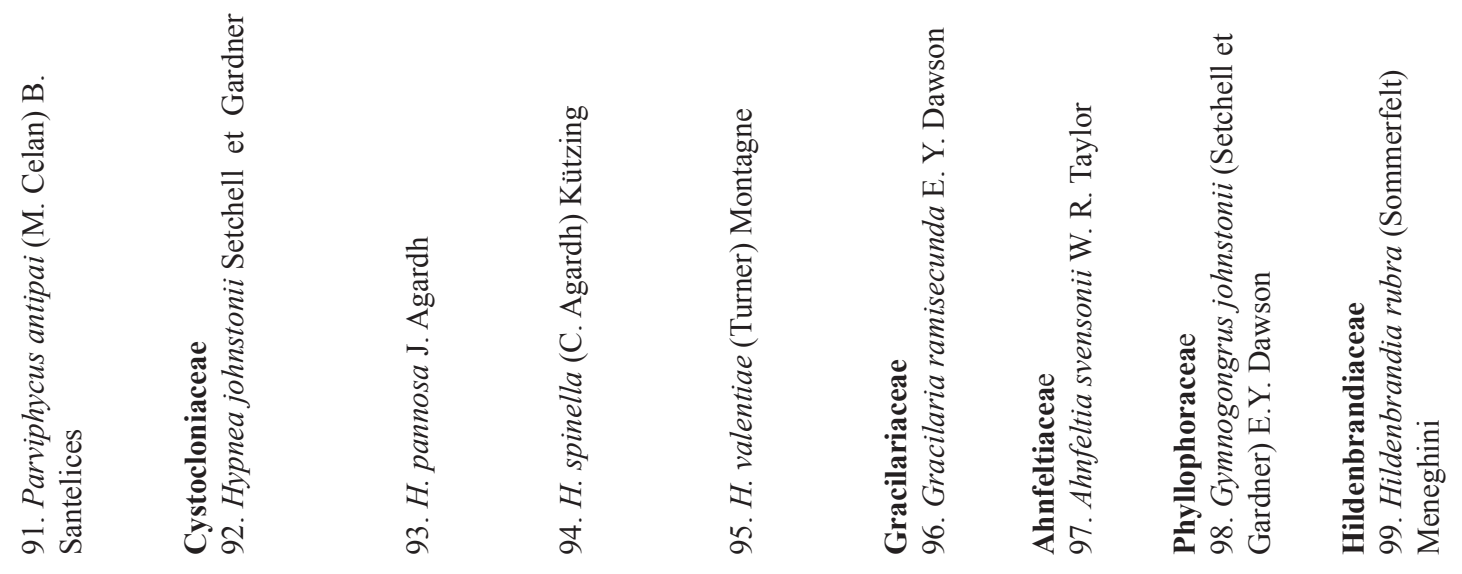




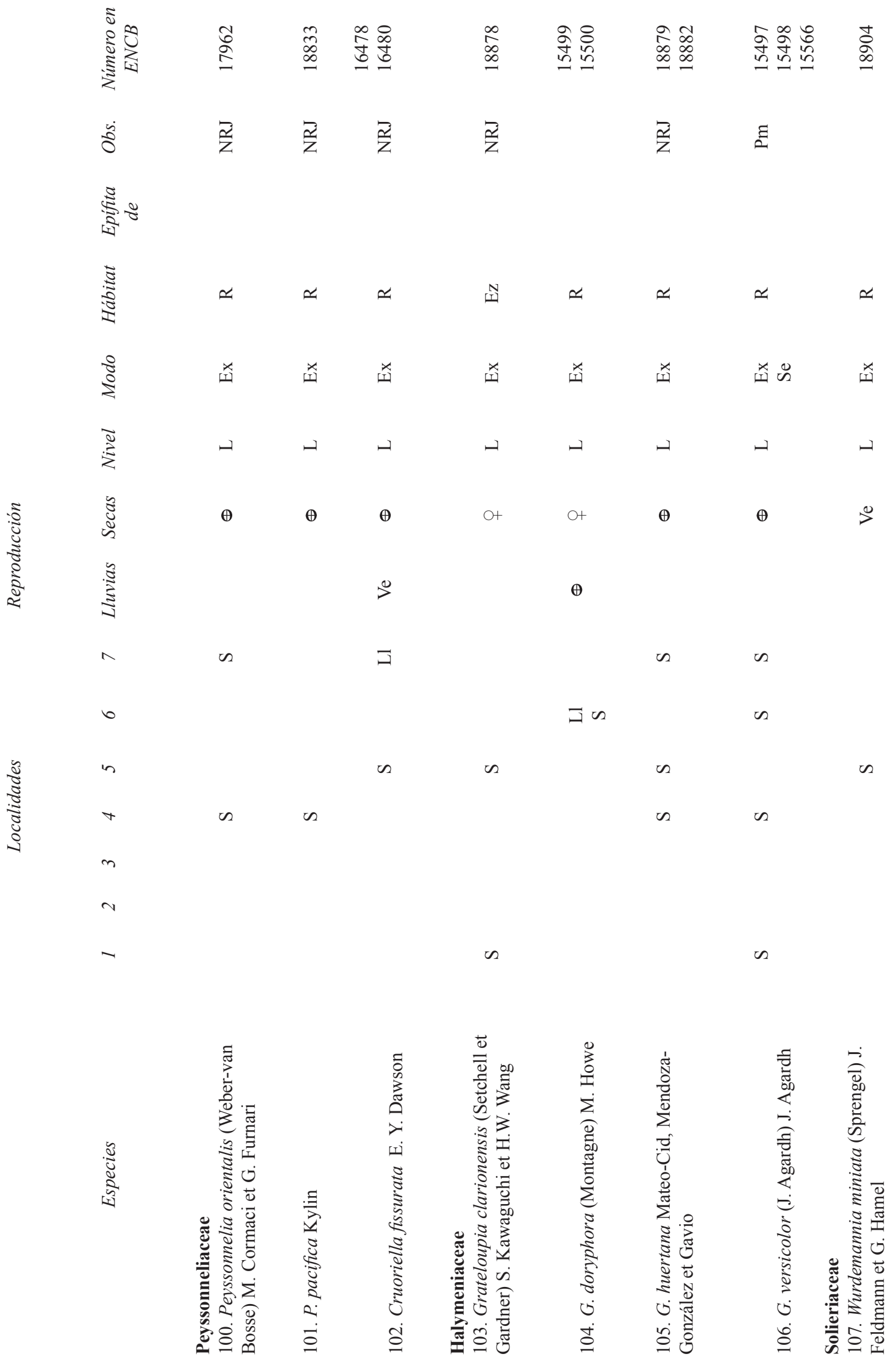




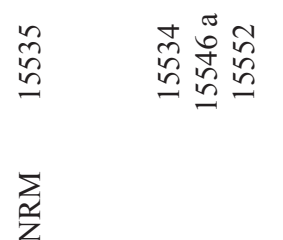

$\stackrel{\infty}{+}$

외시

$\stackrel{\infty}{\infty}$

$\oplus$

1
is
in

屆

盾

$\Theta$

$\Theta$

$\Xi$

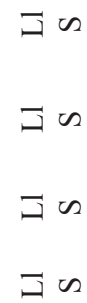

コッ
爷者学

$\cong$

ㄸ 에

$\simeq \quad \simeq$

$\stackrel{5}{a}$

$\Xi \infty$

च

コ

Э
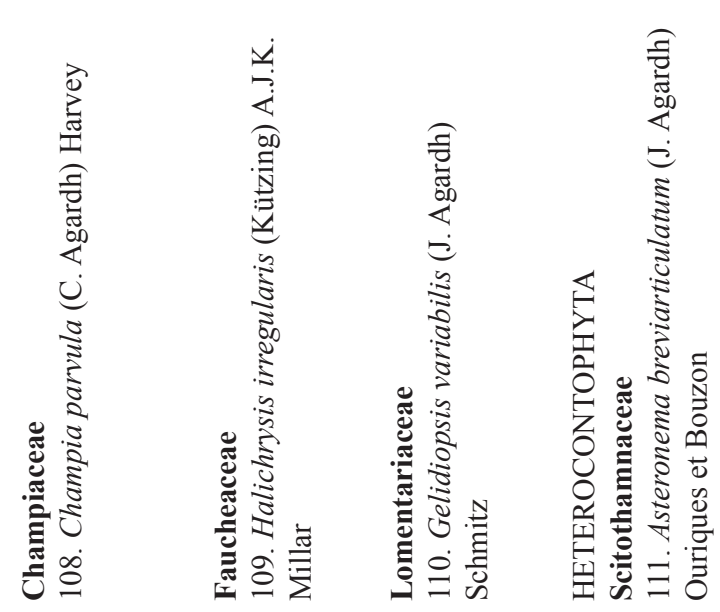

学

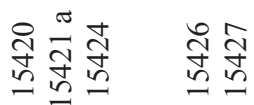

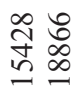

宛

$\widetilde{z}$

学

I

$\frac{3}{2} \quad \frac{3}{2}$

$\frac{3}{2}$

$\frac{3}{2}$

$\frac{7}{2}$

$\frac{3}{2}$

$\frac{3}{2}$

$\frac{3}{2}$

is

コッ コの

is

$\exists \backsim$

$\Xi$

$\exists$

$\Xi$
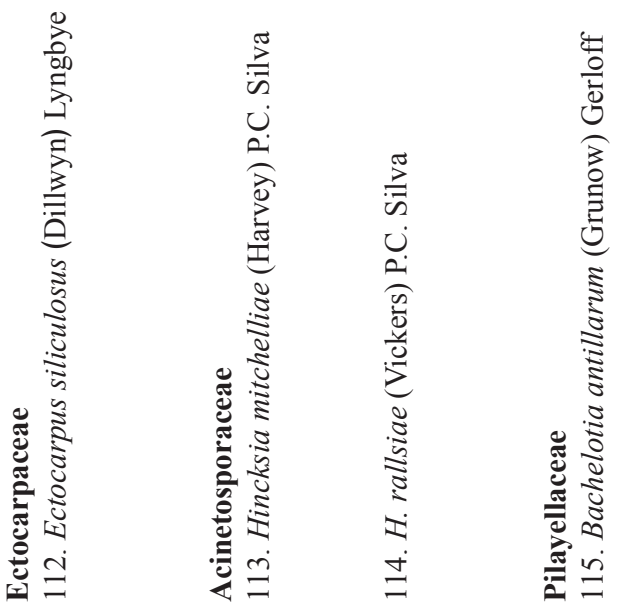


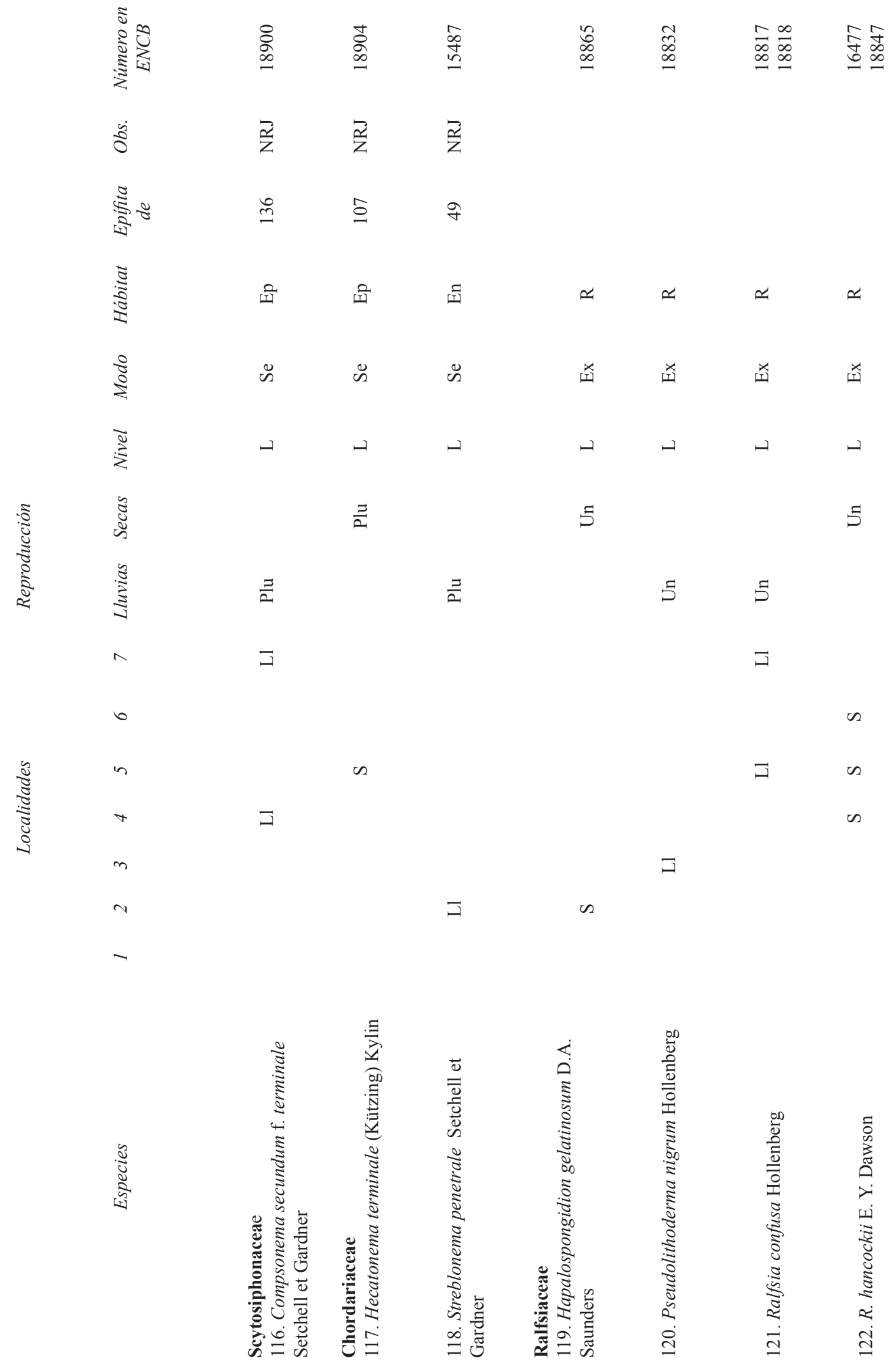




\begin{tabular}{|c|c|c|c|c|c|c|c|c|}
\hline 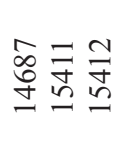 & $\begin{array}{l}\infty \\
\infty \\
\infty \\
\infty\end{array}$ & 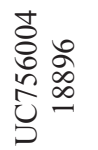 & 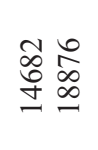 & 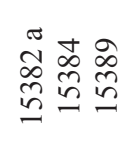 & $\begin{array}{l}\infty \\
\infty \\
\stackrel{\infty}{n} 心 \\
n\end{array}$ & $\stackrel{\check{n}}{\Omega}$ & 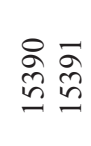 & 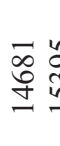 \\
\hline & $\Xi$ & & & $\Xi$ & & & $\Xi$ & \\
\hline & $\hat{n}$ & 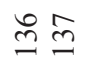 & & & & & & \\
\hline$\simeq$ & ક્વિ & $\simeq$ 외 & $\simeq$ & $\simeq$ & $\simeq$ & $\simeq$ & $\simeq$ & $\simeq$ \\
\hline 差 & 差 & $\ddot{n}$ & 左 & 庄 & 差 & 差 & $\ddot{n}$ & 圼 \\
\hline ــ & ـ & ــ & ــ & ــ & ــ & لـ & ــ & لــ \\
\hline 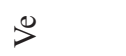 & 2 & 2 & 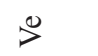 & ot & & & & $>$ \\
\hline 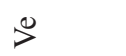 & & & & ot & o+ & o+ & ot & की \\
\hline$\exists \backsim$ & & n & & us & & & & \\
\hline$\Xi$ & is & & & $\Xi$ & $\Xi$ & & $\Xi$ & - \\
\hline & & & is & コ & コ & & & \\
\hline$\exists \infty$ & & & $n$ & is & & & & is \\
\hline$\Xi$ & & & & & $\Xi$ & $\exists$ & $\Xi$ & \\
\hline$\exists \infty$ & & & & & & & & \\
\hline
\end{tabular}
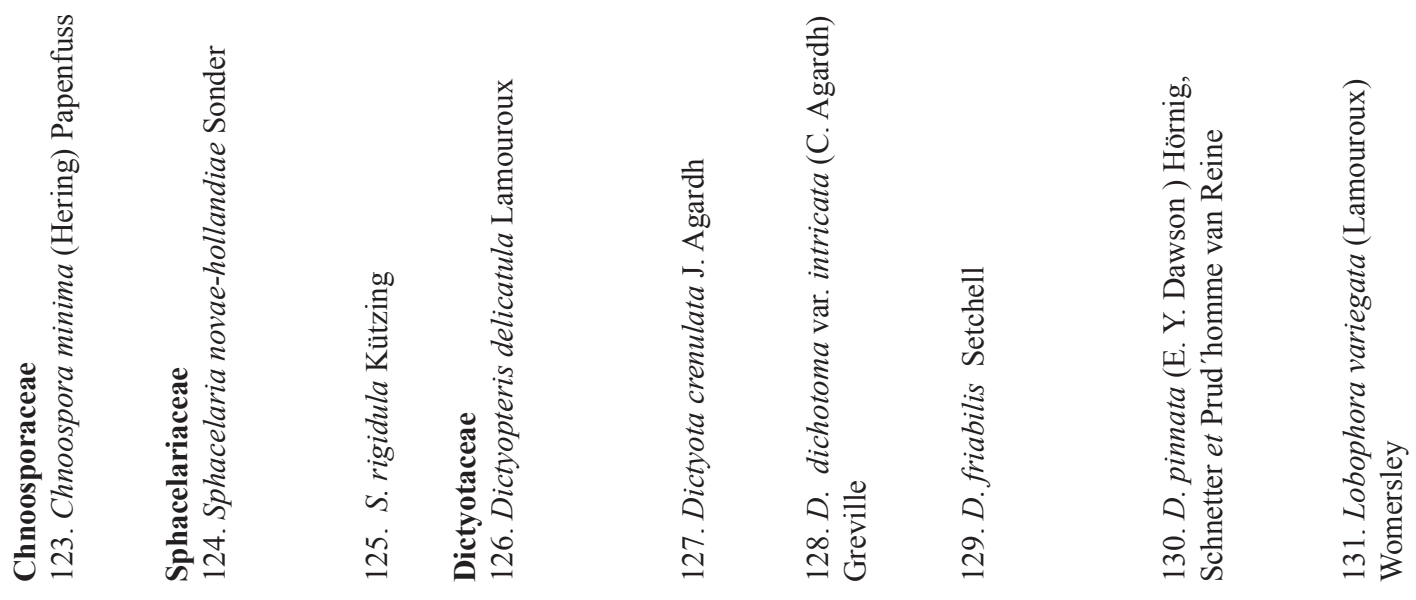


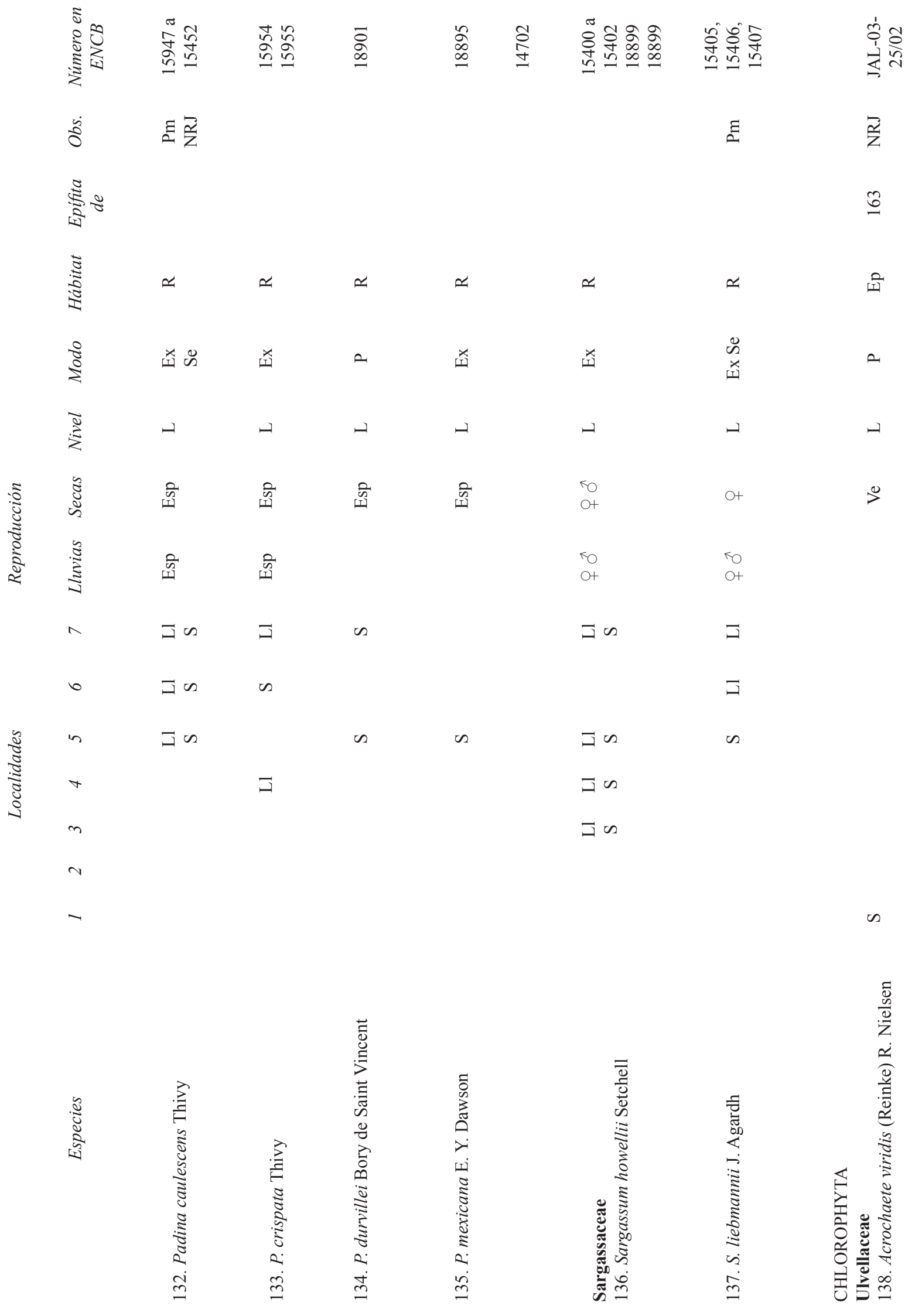




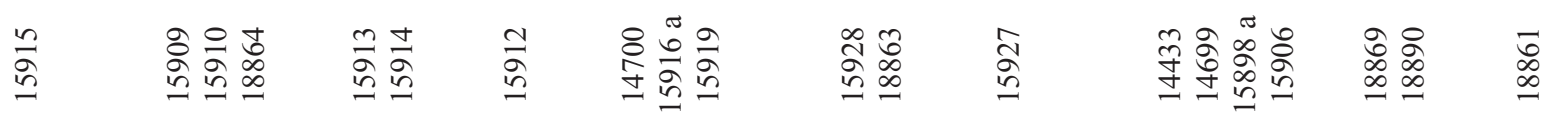

$\Xi$ 믄

$\Xi \quad \vec{\sim}$

$\overrightarrow{\mathrm{z}}$

$\simeq$

$\simeq$

$\simeq$

$\simeq$

$\simeq$

$\simeq$

$\simeq$

$\simeq$

$\simeq$

Nㅗ

爻

肴

$\ddot{n} \times$

压

๗

爻

爻

a

$>$

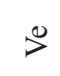

$\stackrel{20}{3}$

$\stackrel{0}{>}$

$>$

s

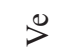

$2 \quad 2$

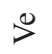

bo

$p^{0}$

2

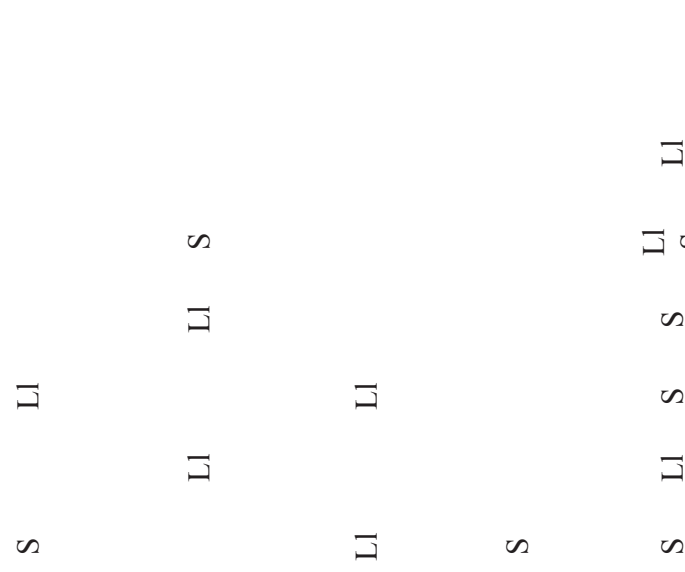

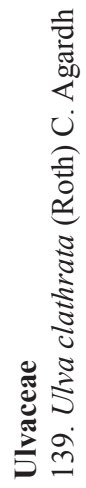
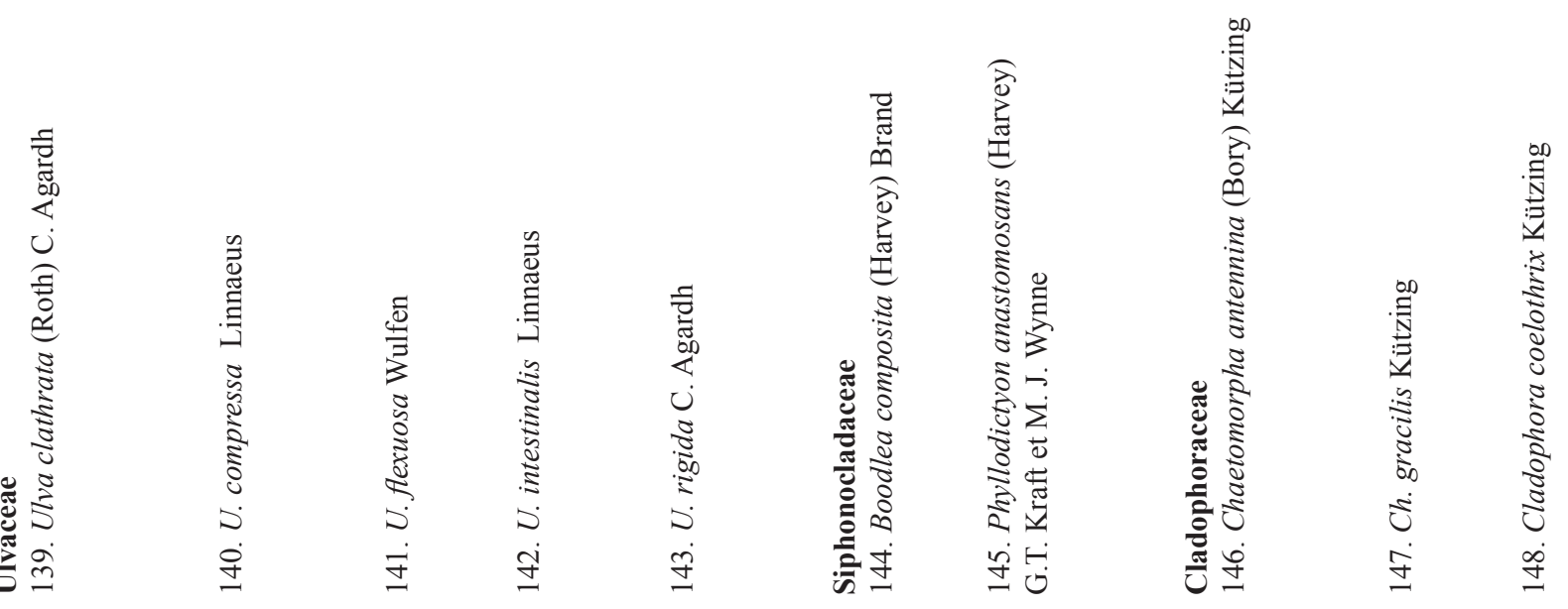

$\frac{n}{3}$

$\infty$ 


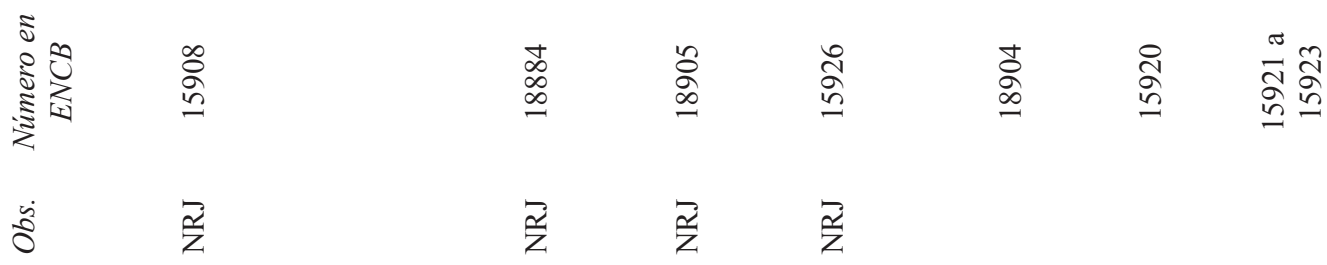

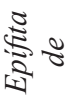

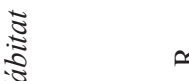

$\simeq$

$\simeq$

$\simeq$

$\simeq$

$\simeq$

$\propto$

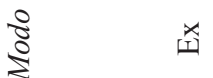

辛

近

$a$

2

I

II

离

$\frac{\bar{z}}{z}$

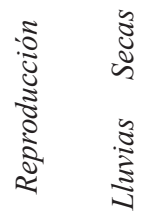

$>$

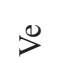

$>$

5

$\stackrel{3}{7}$

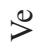

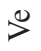

$\nu^{2}$

$\wedge \quad \infty$

6

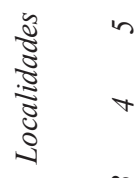

コ

n

コル

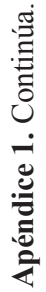

בี

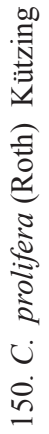

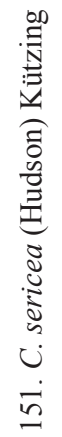

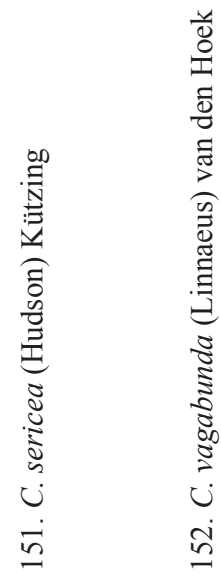

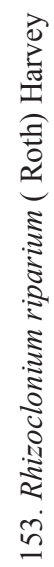

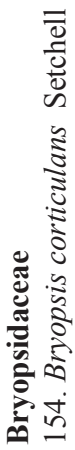

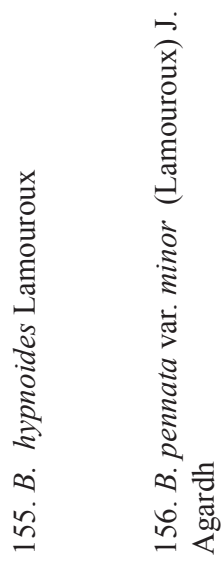




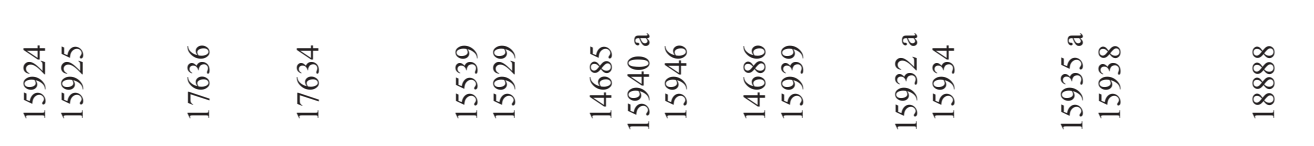

$\Xi$

घ $\quad \overrightarrow{\frac{\pi}{Z}}$

$\Xi$

ह $\frac{\mathrm{z}}{\mathrm{z}}$

$\simeq$

肴

$\approx \approx$

$\simeq \quad \simeq$

$\simeq$

$\simeq$

$\simeq$

肴肴杀

甾甾品杀

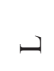

รี

รี

ह

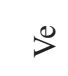

$\times$

is

\lrcorner

घี छ

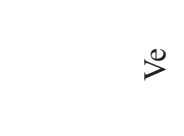

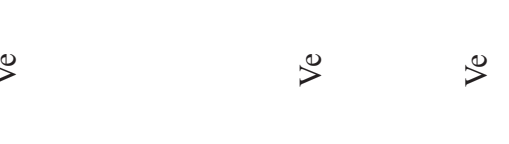

a

In

ヨn $コ$ 的

is
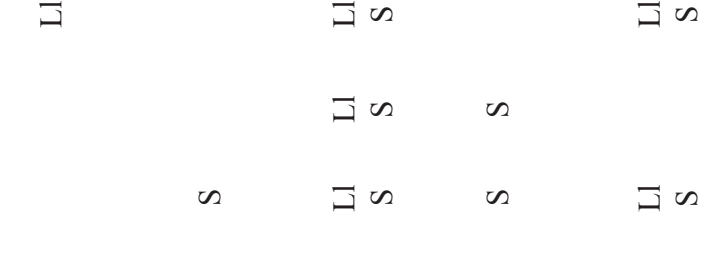

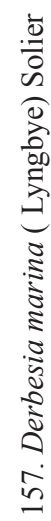
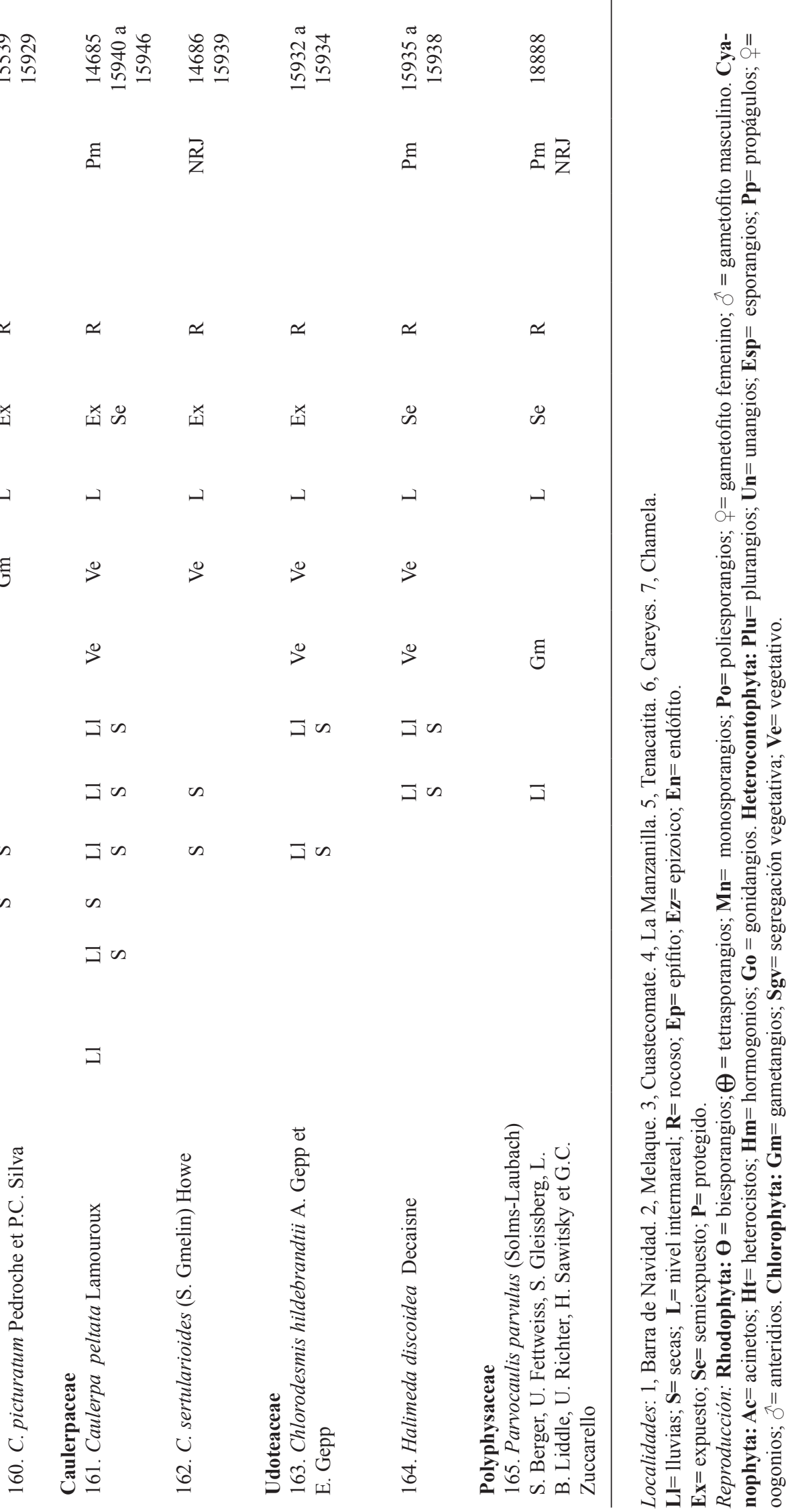
